\title{
A discontinuous quasi upper bound limit analysis approach with sequential linear programming mesh adaptation
}

\author{
G. Milani*1 $^{1}$, P.B.Lourenço ${ }^{+}$ \\ * Department of Structural Engineering (IBK), Swiss Federal Institute of Technology (ETH), \\ Wolfgang-Pauli-Strasse 15, 8093 Zürich, Switzerland \\ ${ }^{+}$Department of Civil Engineering, School of Engineering, University of Minho, Azurém, 4800-058 \\ Guimarães.
}

\begin{abstract}
In this paper, a simple discontinuous upper bound limit analysis approach with sequential linear programming mesh adaptation is presented. Rigid, infinitely strong triangular elements with both linear and Bezier curved edges are considered. A possible jump of velocities is allowed at the interfaces between contiguous elements, thus allowing plastic dissipation on curved interfaces. Bezier curved edges are used with the sole aim of improving the element performance when dealing with limit analysis problems involving curved sliding lines. The model performs poorly for unstructured meshes (i.e. at the initial iteration), being unable to reproduce the typical plastic deformation concentration on inclined slip lines. Therefore, an iterative mesh adaptation based on sequential linear programming is proposed. A simple linearization of the non linear constraints is performed, allowing to treat the NLP problem with consolidated LP routines. The choice of inequalities constraints on elements nodes coordinates turns out to be crucial on the algorithm convergence.
\end{abstract}

Several examples are treated, consisting in the determination of failure loads for ductile, purely cohesive and cohesive frictional materials. The results obtained at the final iteration fit well, for all the cases analyzed, previously presented numerical approaches and analytical predictions.

Keywords: upper bound limit analysis, rigid elements, interfaces, sequential linear programming, associated flow rule, frictionless and frictional materials, masonry

\footnotetext{
${ }^{1}$ Corresponding Author. E-mail: milani@ibk.baug.ethz.ch [Formerly at: University of Ferrara, Department of Engineering, Via Saragat 144100 Ferrara, Italy. Phone: +39 0532 974911. Fax: +39 0532 974870. e-mail: gmilani@ing.unife.it].
} 


\section{Introduction}

The determination of the ultimate load bearing capacity of engineering structures deserves great consideration from a technical point of view. Despite the considerable research efforts done in the past decades in the field of Finite Element (FE) limit analysis, the linear elastic approach is generally considered as the most practical tool to obtain quantitative information for design purposes. Unfortunately, such analysis fails to give an idea of the structural behaviour beyond the beginning of cracking. Especially for cohesive-frictional materials and in the case of masonry, this represents a non-negligible drawback. In fact, due to the relatively low tensile strength of such kind of materials, linear elastic analyses are unable to represent adequately the structural behaviour, even in presence of very low load levels. For this reason, limit analysis is a promising alternative, giving the possibility to predict failure loads and failure mechanisms with a moderate computational effort, requiring only a few mechanical parameters at failure for the simulations. In geomechanics, limit analysis provides a useful method for assessing the load bearing capacity of structures (e.g. footings, retaining walls, etc.) and the stability of slopes and excavations.

While for linear elastic analyses robust and efficient factorization routines are at disposal in FE solvers, for limit analysis only linear programming (LP) routines able to tackle problems involving several variables are needed. Nowadays, commercial LP packages can compete favourably both for stability and time required for the simulations with elastic FE solvers. Furthermore, the time requirements to construct the finite element model are the same as for the elastic analysis.

Nonetheless, limit analysis combines, on one hand, sufficient insight into collapse mechanisms, ultimate stress distributions -at least in critical sections-, and load capacities, and on the other hand, simplicity to be cast into a practical computational tool. Given the difficulties in obtaining reliable experimental data for frictional materials, another appealing feature of limit analysis is the reduced number of necessary material parameters.

Several efforts have been made in this field in the last decades by many authors (see for instance [1]-[7]), with the aim of solving the linear optimization problem by means of non-linear programming routines (NLP), usually avoiding to perform a linearization of the material strength domain. This allowed a further improvement in the numerical efficiency of FE limit analysis programs.

Another important aspect of the FE approach within limit analysis is that the classical lower and upper bound theorems allow to rigorously bound the exact limit load for a perfectly plastic structure. When the bound theorems are implemented numerically in combination with the finite element method, the ability to obtain tight bracketing depends not only on the efficient solution of 
the arising optimization problem, but also on the effectiveness of the elements employed. Elements for (strict) upper bound analysis pose a particular difficulty, since the flow rule is required to hold throughout each element, yet it can only be enforced at a finite number of points. The standard choice for this type of analysis has been the constant strain element combined with discontinuities in the displacement field (see for instance Sloan and Kleeman [1]).

Nevertheless, the accuracy of the approach is highly dependent on the alignment of the discontinuities, meaning that it can perform poorly if an unstructured mesh is employed. Such a poor performance increases when dealing with rigid, infinitely strong elements, as is the case treated here. Plastic dissipation, in this case, occurs only at the interfaces between contiguous elements, thus constraining the collapse loads to be drastically dependent on the disposition of the interfaces in the mesh. In order to circumvent this limitation, re-meshing strategies could be adopted, as suggested in [8]-[9]. In this case, an iterative procedure with increasing number of optimization variables at successive iterations is needed. As an alternative, adaptive upper bound methods with linear elements and possible plastic deformation in both triangles and discontinuities, as proposed in [10], should be used.

Considering the drawbacks related to a triangular discretization, it seems also appealing the generalization (using adaptive schemes at both element and material level) of totally different procedures presented in the recent past in the literature, as for instance the free Galerkin approach (see [11]) or the p-FEM [12].

Differently from existing algorithms available, the basic idea of the procedure proposed here consists in: (a) limiting, as much as possible, optimization variables in order to make the numerical model fast and efficient and (b) reproducing general failure mechanisms involving curved discontinuities, utilizing few elements without dissipation in continuum (and therefore avoiding the introduction of additional plastic multipliers in continuum). Such requirements are somewhat contradictory, since it is well known that linear rigid elements perform well in the reproduction of complex curved collapse mechanisms, only if the mesh utilized is sufficiently refined (i.e. if curved edges, active in the dissipation process, are well approximated by segments).

In this framework, in order to comply accuracy and limited computational effort both a linear and a curved Bezier triangular rigid element with possible dissipation along (curved) interfaces between adjoining elements are presented. Since dissipation can occur only at the interfaces between contiguous elements, a mesh adaptation algorithm able to enforce the shape of the interfaces to coincide with the actual slip lines is adopted. There are many reasons which justify the use of adaptive rigid elements with linear or curved edges. Among the others, the most important ones (which make, indeed, preferable the use of curved elements in some cases) are their ability to 
reproduce complex failure mechanisms with plastic dissipation concentrated on curved slip lines and the simplicity of the algorithm itself, consisting in the recursive utilization of a LP routine. It is worth noting that the utilization of (a) curved elements, (b) relatively coarse meshes and (c) iterative LP schemes differs significantly from adaptive techniques recently presented in the technical literature (see for instance [10][13]), which are usually based on the utilization of NLP codes. The approach here presented, in fact, follows a classical procedure (attempted at the early stages of FE limit analysis research) based on linear programming and linearization of the failure surfaces. Furthermore, it concentrates exclusively on geometrical aspects (linearization of non linear constraints and utilization of splines) rather than on numerical issues related to non-linear programming and therefore may be of interest also for practical engineers not involved in the most recent optimization research.

Bezier curves are treated in the model in the same manner as linear interfaces, with the only difference that more plastic multiplier rates are required for each interface and that plastic dissipation is obtained resorting to numerical integration methods.

For both linear and curved elements, a simple linearization of the non linear constraints is performed, allowing to treat the NLP problem with consolidated LP routines. The choice of inequalities constraints on elements nodes coordinates turns out to be crucial on the algorithm convergence.

Several meaningful examples are treated to validate the procedure proposed, consisting in the determination of failure loads of ductile (plate with central hole), purely cohesive and cohesive frictional materials (indentation problems, masonry shear walls). The results obtained at the final iteration fit well, for all the cases analyzed, previously presented numerical approaches and, where available, analytical predictions.

\section{Triangular upper bound limit analysis}

In this section, an adaptive upper bound limit analysis conducted by means of triangular elements with both linear and curved Bezier edges and possible dissipation between adjoining elements is presented.

Linear elements can be regarded as a particular sub-class of curved elements. Anyway, the reasons at the base of the utilization of adaptive rigid elements with generally curved edges are (1) the capability of the method to reproduce complex failure mechanisms with non linear slip lines more accurately, when compared with standard linear triangles and (2) the simplicity of the algorithm, consisting on a trivial recursive utilization of robust LP routines. For both linear and curved elements, it is worth noting that the method competes favourably with more classical re-meshing 
IJMS-08-37 Revised version R2.

techniques ([8][9]). In fact, while for the present analyses a number of LP problems with the same number of optimization variables has to be solved to converge to suitable solutions, in re-meshing [8] the number of optimization variables increases at successive iterations, meaning that time required to perform the simulation becomes great near the optimal mesh.

\subsection{The linear and the rigid splines element}

Let us consider a triangular element $T$ with curved edges, as shown in Figure 1. Each edge of the element is constituted by cubic Bézier splines defined by four control points $\mathbf{P}_{1}, \mathbf{P}_{2}, \mathbf{P}_{3}$ and $\mathbf{P}_{4}$, see Figure 1-a. As well known from the technical literature [14]-[16], Cartesian coordinates of each point of the Bezier curve can be obtained in parametric form having at disposal $\mathbf{P}_{1} \ldots \mathbf{P}_{4}$ as follows:

$$
\underset{t \in[0,1]}{\mathbf{P}(t)=-(t-1)^{3} \mathbf{P}_{1}+3 t(t-1)^{2} \mathbf{P}_{3}-3 t^{2}(t-1) \mathbf{P}_{4}+t^{3} \mathbf{P}_{2}}
$$

In the model, both a $\mathrm{C} 0$ and $\mathrm{C} 1$ continuity of the edges between adjoining elements can be required. In particular, when a $\mathrm{C} 1$ continuity is required, control points $\mathbf{P}_{3}$ and $\mathbf{P}_{5}$ (see Figure 1-b) in the initial mesh have to be set in such a way that they are aligned with $\mathbf{P}_{4}$. Similar considerations can be repeated for $\mathbf{P}_{6}, \mathbf{P}_{7}$ and $\mathbf{P}_{8}$. During the sequential linear programming mesh adaptation, $\mathrm{C} 1$ continuity requirement yields to a further set of linear equality constraints in the form:

$\mathbf{P}_{4}=\left(\mathbf{P}_{3}+\mathbf{P}_{5}\right) / 2$

Obviously, linear triangular elements are obtained as a sub-class of curved elements simply imposing that:

$$
\begin{aligned}
& \mathbf{P}_{3}=\alpha \mathbf{P}_{1}+(1-\alpha) \mathbf{P}_{2} \\
& \mathbf{P}_{4}=\beta \mathbf{P}_{1}+(1-\beta) \mathbf{P}_{2}
\end{aligned}
$$

with $\alpha, \beta \in\left[\begin{array}{ll}0 & 1\end{array}\right]$.

\subsubsection{Jump of displacement field at the interfaces}

A triangular linear or curved element $T$ with vertices $\mathbf{P}_{1}, \mathbf{P}_{2}$ and $\mathbf{P}_{3}$ and control points $\mathbf{P}_{4} \ldots \mathbf{P}_{9}$ of coordinates $\left(x_{i}, y_{i}\right), i=1, \ldots, 9$ is considered, Figure 2 .

For an arbitrary edge $\Gamma_{i j}^{e}$ of element $T$ connecting nodes $i-j$, a suitable local curved frame of reference $\mathbf{s}^{e}-\mathbf{r}^{e}$ with origin on vertex $i$ and $\mathbf{r}^{e}$ parallel to the interface normal can be easily determined from equation ( 1 ). 
IJMS-08-37 Revised version R2.

Its analytical description in the global frame of reference is necessary in the framework of limit analysis with plastic dissipation allowed only at the interfaces between contiguous elements. The determination of the tangential and normal components with respect to the interface direction of the jump of velocities vector between adjoining elements is, in fact, required for the evaluation of the internal power dissipated.

Let us consider for the sake of conciseness only a 1-2 linear or Bézier curved edge $\Gamma_{12}$, as shown in Figure 2. Similar considerations can be repeated for $1-3$ and 2-3 edges respectively, with no conceptual differences.

From equations ( 1 ) and the total derivative with respect to the independent parameter $t$, the versor $\mathbf{s}^{e}$ can be obtained as follows:

$$
\begin{aligned}
& \mathbf{s}^{e}=\frac{d \mathbf{P}}{d t} /\left\|\frac{d \mathbf{P}}{d t}\right\|=\frac{-3(t-1)^{2} \mathbf{P}_{1}+3\left(3 t^{2}-4 t+1\right) \mathbf{P}_{3}-3\left(3 t^{2}-2 t\right) \mathbf{P}_{4}+3 t^{2} \mathbf{P}_{2}}{\sqrt{\left(-\xi_{1} x_{1}+\xi_{2} x_{3}-\xi_{3} x_{4}+\xi_{4} x_{2}\right)^{2}+\left(-\xi_{1} y_{1}+\xi_{2} y_{3}-\xi_{3} y_{4}+\xi_{4} y_{2}\right)^{2}}} \\
& \mathbf{r}^{e}=\mathbf{s}^{e} \times \mathbf{e}_{3}
\end{aligned}
$$

Where:

$-\xi_{1}=-3(t-1)^{2} \quad \xi_{2}=3\left(3 t^{2}-4 t+1\right) \quad \xi_{3}=-3\left(3 t^{2}-2 t\right) \quad \xi_{4}=3 t^{2}$

- $\mathbf{e}_{3}=\mathbf{e}_{1} \times \mathbf{e}_{2}=\left[\begin{array}{lll}0 & 0 & 1\end{array}\right]$ is the $z$-axis versor.

Therefore, the transformation matrix from the global to the interface local coordinate system reads as follows:

$\left[\begin{array}{l}\mathbf{s}^{e} \\ \mathbf{r}^{e}\end{array}\right]=\left[\begin{array}{ll}\cos \vartheta_{12}(t) & \sin \vartheta_{12}(t) \\ \sin \vartheta_{12}(t) & \cos \vartheta_{12}(t)\end{array}\right]\left[\begin{array}{l}\mathbf{e}_{1} \\ \mathbf{e}_{2}\end{array}\right]=\mathbf{T}(t)\left[\begin{array}{l}\mathbf{e}_{1} \\ \mathbf{e}_{2}\end{array}\right] \Leftrightarrow\left[\begin{array}{l}\mathbf{e}_{1} \\ \mathbf{e}_{2}\end{array}\right]=\mathbf{T}(t)^{-1}\left[\begin{array}{l}\mathbf{s}^{e} \\ \mathbf{r}^{e}\end{array}\right]$

Equation ( 4 ) allows the explicit determination of the components of unit vectors $\mathbf{s}^{e}$ and $\mathbf{r}^{e}$ point by point of the interface. Nevertheless, since edge $1-2$ is a polynomial of degree 3 in $t$, jump of displacements has to be written only in 4 points of the interface in order to describe the field completely for each point of $\Gamma_{12}$.

Furthermore, since the curved triangular elements here adopted are rigid, velocity field interpolation inside each element depends only on 3 independent variables represented respectively by velocities 
$\mathbf{u}_{G}=\left[\begin{array}{ll}u_{x}^{G} & u_{y}^{G}\end{array}\right]^{T}$ of a point of the rigid body and rigid rotation $\boldsymbol{\Phi}_{G}=\left[\begin{array}{lll}0 & 0 & \Phi_{z}^{G}\end{array}\right]^{T}$ along $z$ coordinate axis. For the sake of simplicity, point $\mathbf{G}$ is chosen as the centroid of the triangle connecting points $\mathbf{P}_{1}, \mathbf{P}_{2}, \mathbf{P}_{3}$, see Figure 3 .

Therefore, the velocities' field of the 1-2 edge belonging to a generic element $M$ is expressed in global coordinates as follows:

$\left[\begin{array}{l}u_{x 0}\left(t_{0}\right) \\ u_{y 0}\left(t_{0}\right)\end{array}\right]=\left[\begin{array}{ccc}1 & 0 & \Phi_{z}^{G} \\ 0 & 1 & -\Phi_{z}^{G}\end{array}\right]\left[\begin{array}{l}x_{0}\left(t_{0}\right)-x_{G} \\ y_{0}\left(t_{0}\right)-y_{G}\end{array}\right]=\mathbf{R}_{G}\left(\mathbf{P}_{0}\left(t_{0}\right)-\mathbf{G}\right)$

Where:

- $x_{0}^{r}$ and $y_{0}^{r}$ are global coordinates of a generic point $\mathbf{P}_{0}$ corresponding to $t=t_{0}$ on $\Gamma_{i j}^{e}$ edge.

$-u_{x 0}\left(t_{0}\right)$ and $u_{y 0}\left(t_{0}\right)$ are the horizontal and vertical global velocities of point $\mathbf{P}_{0}$ respectively.

Equation ( 5 ) can be substituted in ( 6 ) in order to obtain the edge velocities' field in the local coordinate system:

$\left[\begin{array}{l}u_{s}(t) \\ u_{r}(t)\end{array}\right]=\mathbf{T}(t)^{-1} \mathbf{R}_{G}(\mathbf{P}(t)-\mathbf{G})$

From the above considerations, when two contiguous elements $M$ and $N$ with common interface $\Gamma_{12}^{e}$ are considered, the jump of displacements at the common interface can be written as:

$\left[\mathbf{u}^{M-N}\right]=\mathbf{T}(t)^{-1}\left[\mathbf{R}_{G}^{M}\left(\mathbf{P}(t)-\mathbf{G}^{M}\right)-\mathbf{R}_{G}^{N}\left(\mathbf{P}(t)-\mathbf{G}^{N}\right)\right]$

with obvious meaning of all the symbols introduced.

\subsubsection{Plastic flow relationships and power dissipation}

From equation ( 7 ), power dissipated at the interfaces can be numerically evaluated in the local coordinate system. With this aim, the stress vector $\mathbf{t}^{I}$ acting at the interface $I$ between contiguous elements is introduced, Figure 4, defined as $\mathbf{t}^{I}=\left[\begin{array}{ll}\tau_{s}^{I} & \sigma_{r}^{I}\end{array}\right]^{T}$ and constituted by a normal stress $\sigma_{r}^{I}$ acting along local axis $\mathbf{r}^{e}$ and a tangential stress $\tau_{s}^{I}$ acting along $\mathbf{s}^{e}$ respectively. 
IJMS-08-37 Revised version R2.

On the other hand, the length of the edges can be evaluated by means of a numerical integration as follows:

$$
\Gamma_{i j}^{e}=\int_{0}^{1} d s^{e}=\int_{0}^{1} \sqrt{(d y / d t)^{2}+(d x / d t)^{2}} d t
$$

From the above considerations, power dissipated at the interface is evaluated solving analytically the following integral:

$$
P^{I}=\int_{0}^{1}\left(\sigma_{s}^{I}\left[u_{s}^{M-N}\right]+\tau_{r}^{I}\left[u_{r}^{M-N}\right]\right) \sqrt{(d x / d t)^{2}+(d y / d t)^{2}} d t
$$

For each interface $I$ of length $L_{12}$, we suppose to have at disposal a linearized strength domain for the material constituting the interface and defined by $m^{I}$ planes in the local coordinate system (of equation $A_{s}^{q^{I}} \sigma_{s}^{I}+A_{r}^{q^{I}} \tau_{r}^{I}=C_{I}^{q^{I}} 1 \leq q^{I} \leq m^{I}$ ). Introducing plastic multipliers fields at the interface (one for each linearization plane), the well know associated plasticity relation can be written:

$$
\begin{aligned}
& {\left[u_{s}^{M-N}\right]=\sum_{q^{I}}^{m^{I}} \dot{\lambda}_{q^{I}}^{I}(t) A_{s}^{q^{I}}} \\
& {\left[u_{r}^{M-N}\right]=\sum_{q^{I}}^{m^{I}} \dot{\lambda}_{q^{I}}^{I}(t) A_{r}^{q^{I}}}
\end{aligned}
$$

where $\dot{\lambda}_{q^{I}}^{I}(t)$ represents the $q^{\text {th }}$ plastic multiplier rate field.

It is stressed that, when dealing with problems involving friction (as in the case, for instance, of geotechnical problems and masonry structures), the current approach still assumes classical normality (otherwise, as well known, the bound theorems of limit analysis are inapplicable and a different mathematical formulation should be adopted, see Ferris and Tin-Loi [17]).

It is worth noting that, when curved triangular elements are considered, equation ( 11 ) is weakened under the form of inequality, in order to avoid that algorithm fails to find a solution (as underlined, for instance, in [5]-[7]). In particular, a small tolerance TOL is introduced so that: 


$$
\begin{aligned}
& \sum_{q^{I}}^{m^{I}} \dot{\lambda}_{q^{I}}^{I}(t) A_{s}^{q^{I}}-T O L<\left[u_{s}^{M-N}\right]<\sum_{q^{I}}^{m^{I}} \dot{\lambda}_{q^{I}}^{I}(t) A_{s}^{q^{I}}+T O L \\
& \sum_{q^{I}}^{m^{I}} \dot{\lambda}_{q^{I}}^{I}(t) A_{r}^{q^{I}}-T O L<\left[u_{r}^{M-N}\right]<\sum_{q^{I}}^{m^{I}} \dot{\lambda}_{q^{I}}^{I}(t) A_{r}^{q^{I}}+T O L
\end{aligned}
$$

Parameter TOL in the previous equation is set in the simulations equal to $\chi\left[u_{s}^{M-N}\right]$, with $\chi$ small scalar constant. As a rule, $\chi$ has been chosen in the range 1/50-1/200. Authors experienced that stability of the algorithm is optimal for $\chi$ around $1 / 80$, but this parameter has to be tuned case by case by the user.

In what follows, curvature of the edges is always small if compared with linear elements, therefore the introduction of equation ( 12 ) permit a sufficient stability of the algorithm which otherwise could fail (Makrodimopoulos and Martin [5]) in presence of jump of displacements different from a simplex. Obviously, using equation ( 12 ), the property of obtaining strict upper bound approximations for the collapse load is lost; nonetheless, the approximation introduced results in good estimations (at least from an engineering point of view) of failure loads, as shown in the last section. Such an approximation is avoided when linear elements are used.

In this way, from equations $(10)(11)$, the power dissipated at the interface can be re-written as:

$$
\begin{aligned}
& P^{I}=\int_{0}^{1} \sum_{q^{I}}^{m^{I}} \dot{\lambda}_{q^{I}}^{I}(t)\left[A_{s}^{q^{I}} \sigma_{s}^{I}+A_{r}^{q^{I}} \tau_{r}^{I}\right] \sqrt{(d x / d t)^{2}+(d y / d t)^{2}} d t= \\
& =\int_{0}^{1} \sum_{q^{I}}^{m^{I}} \dot{\lambda}_{q^{I}}^{I}(t) C_{I}^{q^{I}} \sqrt{(d x / d t)^{2}+(d y / d t)^{2}} d t
\end{aligned}
$$

Obviously, $\dot{\lambda}_{q^{I}}^{I}(t)$ field should assume the same analytical expression found for the jump of velocity at the interface, equation ( 7 ). The analytical determination of $\dot{\lambda}_{q^{I}}^{I}(t)$ field is not a trivial task and is not strictly necessary from a numerical point of view. Furthermore, a limitation of the number of optimization variables for each interface is always desirable. Here, for the sake of simplicity, a cubic polynomial in $t$ both for plastic multipliers and for the jump of velocities field is assumed (see Figure 5). Therefore, the $\dot{\lambda}_{q^{I}}^{I}(t)$ field is fully determined introducing only 4 plastic multipliers 
IJMS-08-37 Revised version R2.

for each linearization plane for each internal, corresponding to $t=0,1 / 3,2 / 3$ and 1 respectively. In

this way, plastic dissipation at the interface can be handled numerically with a small computation effort as follows:

$$
P^{I}=\sum_{q^{I}}^{m^{I}}\left(W_{1} \dot{\lambda}_{q^{I}}^{I, 1}+W_{3} \dot{\lambda}_{q^{I}}^{I, 3}+W_{4} \dot{\lambda}_{q^{I}}^{I, 4}+W_{2} \dot{\lambda}_{q^{I}}^{I, 2}\right) C_{I}^{q^{I}}
$$

where:

- $W_{i} i=1,2,3,4$ are numerical coefficients easily determined by the authors making use of a subroutine which operates on a generic curved interface by means of the Symbolic Matlab toolbox [18]. In particular, assuming $\dot{\lambda}_{q^{I}}^{I}(t)=a_{0}+a_{1} t+a_{2} t^{2}+a_{3} t^{3}$, constants $a_{i} i=0,1,2,3$ are determined symbolically imposing that $\dot{\lambda}_{q^{\prime}}^{I}(t)=\lambda_{q^{\prime}}^{I, i}$. It is worth noting that constants $a_{i} i=0,1,2,3$ result linear functions in $\lambda_{q^{I}}^{I, i}$. Hence $\int_{0}^{1} \sum_{q^{I}}^{m^{I}} \dot{\lambda}_{q^{I}}^{I}(t) C_{I}^{q^{I}} \sqrt{(d x / d t)^{2}+(d y / d t)^{2}} d t$ is symbolically evaluated, leading to the numerical determination of $W_{i}$ constants;

- $\dot{\lambda}_{q^{\prime}}^{l, i}$ is the plastic multiplier of node $i$.

External power dissipated can be written as $P^{e x}=\left(\mathbf{P}_{0}^{T}+\lambda \mathbf{P}_{1}^{T}\right) \mathbf{w}$, where $\mathbf{P}_{0}$ is the vector of (equivalent lumped) permanent loads, $\lambda$ is the load multiplier for the structure examined, $\mathbf{P}_{1}^{T}$ is the vector of (lumped) variable loads and $\mathbf{w}$ is the vector of assembled nodal velocities. As the amplitude of the failure mechanism is arbitrary, a further normalization condition $\mathbf{P}_{1}^{T} \mathbf{w}=1$ is usually introduced. Hence, the external power becomes linear in $\mathbf{w}$ and $\lambda$.

\subsubsection{The Linear Programming (LP) problem}

After some elementary assemblage operations, a simple linear programming problem is obtained (analogous to the dual or primal problem reported in [19]-[22]), where the objective function consists in the minimization of the total internal power dissipated: 


$$
\left\{\begin{array}{l}
\min \left\{\sum_{I=1}^{n^{I}} P_{I}^{i n}\left(\dot{\lambda}^{I, a s s}\right)-\mathbf{P}_{0}^{T}\left[\begin{array}{ll}
\mathbf{w} & \boldsymbol{\Phi}
\end{array}\right]^{T}\right\} \\
\text { such that }\left\{\begin{array}{l}
\mathbf{A}^{e q} \mathbf{U}=\mathbf{b}^{e q} \\
\dot{\lambda}^{I, a s s} \geq \mathbf{0}
\end{array}\right.
\end{array}\right.
$$

where:

- $\quad \mathbf{U}$ is the vector of global unknowns and collects the vector of elements centroids velocities ( $\mathbf{w}$ ) and rotation rates $(\boldsymbol{\Phi})$ and the vector of assembled interface plastic multiplier rates $\left(\dot{\lambda}^{\text {I,ass }}\right)$.

- $\quad \mathbf{A}^{e q}$ is the overall constraints matrix and collects normalization conditions, velocity boundary conditions, constraints for plastic flow in velocity discontinuities and compatibility conditions.

- $\quad n^{I}$ is the total number of interfaces in the model.

The reader is referred to [19]-[22] for a critical discussion of the most efficient tools for solving the upper bound (primal or dual) linear programming problem reported in equation ( 15 ).

\subsection{Mesh adaptation by means of a sequential linear programming scheme and SLP}

In this section, a sequential linear programming scheme is presented which allows the adaptation at each step of the mesh used. The aim is of reproducing real failure mechanisms of 2D limit analysis problems as closest as possible with the numerical approach proposed, even with relatively coarse discretizations. If variation in an element geometry is considered, equality constraints $(8)(11)(14$ ) become non linear. The technique of sequential linear programming (SLP) relies on an iterative process in which linearized approximations are used in any particular iteration [23]-[25]. It is worth mentioning that SLP schemes have been adopted by many authors in the recent past for the limit analysis of slabs, see for instance Johnson [23] [25] and Ramsay and Johnson [24] making use of triangular linear elements. While triangular elements with linear edges are adequate for out-of-plane problems as shown by Johansen [26], such an approach may give an overestimation of the actual collapse loads in the case of in-plane problems, due to the fact that slip lines are usually non linear. 
IJMS-08-37 Revised version R2.

Therefore, a limit analysis approach with both linear and Bèzier triangular elements may be useful, as is the case here treated.

In order to use a sequential linear programming scheme, coordinates of control points of each interface have to be taken as optimization variables. Each non linear inequality and/or equality constraint may be linearised by replacing each term by its first-order Taylor series approximation.

For instance, a first order approximation of equation ( 8 ) becomes:

$\left.\sum_{q^{I}}^{m^{I}} \dot{\lambda}_{q^{I}}^{I}(t)\right|_{i-1}\left[\begin{array}{c}A_{s}^{q^{I}} \\ A_{r}^{q^{I}}\end{array}\right]-\mathbf{T}(t)_{i-1}^{-1}\left[\left.\mathbf{R}_{G}^{M}\right|_{i-1}\left(\left.\mathbf{P}(t)\right|_{i-1}-\left.\mathbf{G}^{M}\right|_{i-1}\right)-\left.\mathbf{R}_{G}^{N}\right|_{i-1}\left(\left.\mathbf{P}(t)\right|_{i-1}-\left.\mathbf{G}^{N}\right|_{i-1}\right)\right]+\left.\nabla \mathbf{f}^{\mathbf{u}^{M-N}}\right|_{i-1}\left(\mathbf{X}_{i}-\mathbf{X}_{i-1}\right)$

where:

- Indices $i$ and $i-1$ represent the actual and the previous iteration in the optimization process respectively;

$-\left.\quad \nabla \mathbf{f}^{\mathbf{u}^{M-N}}\right|_{i-1}$ is the gradient of equation ( 8 );

- $\quad \mathbf{X}_{i}$ and $\mathbf{X}_{i-1}$ collect all optimization variables (i.e. horizontal $\mathbf{U}^{E}$ and vertical $\mathbf{V}^{E}$ elements velocities, elements rotations $\boldsymbol{\Phi}^{E}$, interfaces plastic multipliers $\dot{\lambda}^{I}$ and nodes coordinates $\mathbf{x}^{N}$, $\left.\mathbf{y}^{N}\right)$ in the $i$-th and $(i-1)$-th iteration.

Analogously, a linearization of equation ( 14 ) can be written as follows:

$$
\left.P^{I}\right|_{i}=\sum_{q^{I}}^{m^{I}}\left(\left(W_{1} \dot{\lambda}_{q^{I}}^{I, 1}\right)_{i-1}+\left.\left(W_{3} \dot{\lambda}_{q^{I}}^{I, 3}\right)\right|_{i-1}+\left(W_{4} \dot{\lambda}_{q^{I}}^{I, 4}\right)_{i-1}+\left(W_{2} \dot{\lambda}_{q^{I}}^{I, 2}\right) \|_{i-1}\right) C_{I}^{q^{I}}+\left.\nabla P^{I}\right|_{i-1}\left(\mathbf{X}_{i}-\mathbf{X}_{i-1}\right)
$$

Where $\left.\nabla P^{I}\right|_{i-1}$ is the gradient of equation ( 14 ) and all the other symbols have been already introduced.

Equations ( 16 ) and ( 17 ) replace classic linear programming constraints ( 8 ) ( 14 ) and the linear programming solution is repeated until a desired adjustment of the initial mesh is reached. At the conclusion of each linear programming iteration, the nodes coordinate values $\mathbf{x}^{N}$ and $\mathbf{y}^{N}$ will indicate directly the mesh adaptation. 
IJMS-08-37 Revised version R2.

Obviously, the magnitude of the changes to the geometric variables cannot be obtained from a linear solution and must be enforced by the imposition of suitable inequality bounds. The geometric variables will need to be constrained by lower and upper bounds as follows:

$\left\{\begin{array}{l}\mathbf{x}^{-} \leq \mathbf{x}_{i}^{N} \leq \mathbf{x}^{+} \\ \mathbf{y}^{-} \leq \mathbf{y}_{i}^{N} \leq \mathbf{y}^{+}\end{array}\right.$

where:

$-\mathbf{x}^{-}, \mathbf{y}^{-}, \mathbf{x}^{+}, \mathbf{y}^{+}$are respectively lower and bound vectors for $x$ and $y$ nodes coordinates;

- $\mathbf{x}_{i}^{N}$ and $\mathbf{y}_{i}^{N}$ are horizontal and vertical vectors of nodes coordinates at the $i$-th iteration.

For particular nodes with coordinates $x_{F}^{N}$ and $y_{F}^{N}$, it is required that their position is not altered, to ensure that the boundaries of the problem and its basic topology are not changed. This implies that, e.g. $x_{F}^{-}=x_{F}^{N}=x_{F}^{+}$and $y_{F}^{-}=y_{F}^{N}=y_{F}^{+}$. Nevertheless, from a numerical point of view it is preferable to avoid the use of inequalities ( 18 ) as strict equalities (i.e. $x_{F}^{-}=x_{F}^{N}=x_{F}^{+}$). In these cases, in fact, it is preferable (to ensure stability of the algorithm) to remove $x_{F}^{N}$ and $y_{F}^{N}$ from optimization variables.

Finally, since failure load of the linearized linear programming problem does not necessarily represent a rigorous upper bound of the true collapse load, a post-processing linear programming problem with the new positions of nodes obtained from the $i$-th iteration kept fixed has to be performed. The pseudo-code of the SLP algorithm used for all the examples treated is shown in Figure 6.

\subsection{Nodes condensation at successive iterations}

Condensation of nodes with re-meshing may be necessary passing from one iteration to the successive, in order to avoid topological errors (i.e. overlapping of existing elements). In particular, at the end of each iteration, overlapping of elements is checked with a double strategy. The first strategy consists in measuring the distance between each node and its neighbors: if a limit lower bound distance $r_{\text {lim }}$ is surpassed between two nodes, Figure 7, nodes are condensed into one and a 
IJMS-08-37 Revised version R2.

re-meshing is performed. As a rule, if bounds on nodes positions at each iteration (i.e. vectors $\mathbf{x}^{-}$, $\mathbf{y}^{-}, \mathbf{x}^{+}, \mathbf{y}^{+}$) are suitably set (typically $1 / 10$ of elements edge), such a preliminary procedure precludes the program to commit frequently topological errors. Nevertheless, in order to make the algorithm more robust, a second strategy, computationally more onerous, is performed at the end of each iteration, consisting in selecting with a simple Monte Carlo strategy a fixed number of points internal to the splines triangle under consideration (the procedure is much more easier for linear elements) and in checking if each of the points so selected results external to all the neighboring triangles. A semi automatic control is in any case performed at the end of each iteration, in which the topology correctness is manually assessed.

It is interesting to notice that such a procedure is handled exchanging automatically data from Matlab [18] to a FE mesh generator within Strand 7 FE software [27]. In particular, the new mesh at each iteration is generated by Strand 7 mesh generator, whereas each topological correction is checked manually before passing data to Matlab processor.

\section{Numerical examples}

In this section, the performance of the adaptive upper bound formulation presented is assessed by using it to predict the collapse load of a number of technically meaningful examples. In particular, at a first attempt, the capabilities of the model are assessed on a simple uniaxial tension test on a square plate with central circular hole. The exact collapse solution for a Tresca failure criterion is well known (see for instance Chen and Han [28]) and is characterized by an in-plane symmetrical shearing failure mechanism. In this case, the failure mechanism is determined by a diagonal straight line, therefore splines and linear elements provide the same failure load at the converged solution. An initial distorted mesh is used in order to test the capabilities of the sequential procedure proposed. The second example relies on a plane strip footing (see also [29]) both with frictionless and frictional material. In both cases, the exact collapse load is known. The third analysis, consisting in the evaluation of the failure load of an ice sheet obeying the well known Reinicke and 
IJMS-08-37 Revised version R2.

Ralston [30] failure criterion and subjected to indentation is aimed at testing the capabilities of the procedure in presence of a non-linear failure criterion for the interfaces. A piecewise linear approximation of it is used in the simulations, meaning that several plastic multipliers for each interface are introduced. The last example is a masonry shear wall experimentally tested at ETH Zurich by Ganz and Thurlimann [31]. When dealing with the shear wall, numerical results are obtained assuming for masonry a homogenized linearized failure criterion $S^{\text {hom }} . S^{\text {hom }}$ is obtained making use of a equilibrated micro-mechanical model recently presented in the technical literature [32].

From the results of the numerical simulations reported, it is possible to conclude that the adaptive sequential linear programming scheme allows to approximate rather well analytical solutions, in terms of collapse load and failure mechanism, meaning that the approach proposed is able to provide accurate upper bounds.

\subsection{Square plate with central circular hole}

The first example is a plane stress problem consisting of a square plate of edge length $L$ with a central circular hole of diameter $d$ and subjected to uniaxial tension, as shown in Figure 8. For the problem at hand, a $d / L$ ratio equal to 0.2 is assumed, in agreement with numerical simulations performed by $\mathrm{Yu}$ and Tin-Loi in [33]. Let $\sigma_{0}$ be the uniaxial tensile strength of the specimen, obeying a Tresca failure criterion in plane stress, and $p$ the failure pressure, as indicate in Figure 8. A distorted initial mesh (only $1 / 4$ of the plate is modelled for symmetry, Figure 9) is used both for the linear and splines triangular element. Since plastic dissipation occurs only at the interfaces between adjoining elements, the true inclined sliding line is not included in the possible failure mechanisms reproducible with the initial mesh. Failure mechanisms obtained at the first and last iteration are shown in Figure 9 and Figure 10 respectively. As a consequence of the fact that the initial mesh does not contain the true slip line of the model at hand, an overestimation of $11.25 \%$ of the true collapse load is obtained at the initial iteration, both for the linear triangular and the splines 
IJMS-08-37 Revised version R2.

triangular element, as depicted in Figure 11, where the collapse load at successive iterations is shown for both models. As it is possible to notice, convergence is almost identical for both models, occurring in practice after the sixth iteration. The adaptation of the mesh performed with the algorithm proposed allows the reproduction of the true failure mechanism, which consists in a $45^{\circ}$ inclined yield departing from the bottom left corner of the mesh, as show in Figure 10.

\subsection{Strip footing problem}

Two different examples are treated in this section, namely a strip footing on a frictionless soil and a strip footing on a cohesive-frictional material. In both cases, analytical formulas are at disposal to determine the true collapse load. In what follows, it is shown how the mesh adaptation allows to obtain collapse loads in good agreement with analytical predictions.

\subsubsection{Case I: frictionless soil}

The exact collapse pressure $q_{f}$ of a smooth strip footing on a purely cohesive soil is given by the well known expression $q_{f}=N_{c} c_{u}$, where $c_{u}$ is the undrained shear strength and the bearing capacity factor is $N_{c}=2+\pi(5.14)$.

Two different meshes are used to analyze the problem at hand, see Figure 12. The first, here denoted as M1, is used only for the three noded model, whereas the second (denoted as M2) is utilized both for the linear and the curved elements approach. Such a comparison is reported in order to show that curved elements are able to provide better results in terms of collapse load when compared with linear elements with almost the same number of optimization variables involved.

The results obtained with both meshes in terms of both $N_{c}$ and failure mechanisms are reported at successive iterations from Figure 13 to Figure 15.

As can be noted from Figure 13, the real collapse load of the strip footing is very well approximated using curved elements, with a percentage error at the converged iteration of only $3.31 \%$. It is 
IJMS-08-37 Revised version R2.

interesting to note that the initial iteration furnishes the same collapse load, which strongly overestimate the actual value of $N_{c}$, with a percentage error equal to $21.56 \%$.

In Figure 14 the failure mechanism obtained by means of mesh M1 at successive iterations is reported. As one can note, the adaptation at the converged iteration is non optimal; in particular, the region of soil involved by the failure mechanism is slightly overestimated, meaning that mesh used is too coarse to reproduce accurately the real behavior of the soil.

Such an overestimation is eliminated when mesh M2 is used, as reported in Figure 15, where meshes and failure mechanisms at the converged iteration for both linear and curved elements is reported.

\subsubsection{Case II: soil with friction}

The Prandtl collapse pressure for a surface footing on a weightless cohesive-frictional material is $q_{f}=N_{c} c^{\prime}$ where $N_{c}=e^{\pi \tan \phi^{\prime}}\left[\tan ^{2}\left(\frac{\pi}{4}+\frac{\phi^{\prime}}{2}\right)-1\right] \cot \phi^{\prime}$ and $\phi^{\prime}$ and $c^{\prime}$ are the effective friction angle and cohesion respectively.

For the example at hand, only the mesh shown in Figure 16 is used for the simulations, representing a quite refined discretization. As in the previous case, both linear triangular and Bezier elements are used and compared.

A Mohr-Coulomb failure criterion is assumed for the soil with $\phi^{\prime}=30^{\circ}$ and $c^{\prime}=1 \mathrm{MPa}$. In this case, the Prandtl exact value assumed by constant $N_{c}$ is 30.14 . The results in terms of $N_{c}$ at successive iterations obtained with both models are reported at successive iterations in Figure 17.

As one can note from the successive values of $N_{c}$, the real collapse load of the strip footing is very well approximated using both curved and linear elements, with a percentage error at the converged iteration of only $2.30 \%$. Finally it is interesting to underline that the result at the first iteration strongly overestimates the actual value of $N_{c}$, with a percentage error equal to $126 \%$. In any case, 
IJMS-08-37 Revised version R2.

such an error is comparable with those committed using rigid-plastic linear triangular elements without discontinuities of the velocity field (see [1]).

In Figure 18, failure mechanisms obtained with the initial and final mesh (curved elements) are reported. As it is possible to notice, the final mesh approximates very well the analytical failure mechanism suggested by Prandtl, which consists of a linear triangular element which translates vertically after the point of application of the load, followed by a processing zone with a curved shape line (logarithmic spiral shape), ending with a rigid isosceles triangle moving vertically and horizontally. Since plastic dissipation occurs only at the interface between contiguous elements, it is easily justifiable the inaccurate result obtained with the initial mesh, which is obviously unable to reproduce the true collapse mechanism. Furthermore, it is worth noting that, in this case, the curved element gives, at fixed iteration, better results with respect to the linear one, being the true failure mechanism due to the formation of non-linear slip lines, Figure 17.

\subsection{Ice indentation problem}

The problem of the indentation of an ice sheet, Figure 19, is of engineering interest because of its similarity to the crushing of ice sheets moving towards a vertical pier. If the indentator is assumed prismatic with width $b$, as indicated in Figure 19, the problem may be idealized, depending on the $b / t$ ratio as a either plane strain $(b / t=0)$ or plane stress $(b / t=\infty)$ problem. For the sake of conciseness, in the following only the plane strain case is taken into consideration, being the plane stress problem conceptually similar.

As already discussed by Reinicke and Ralston [30], see also Krabbenhoft et al. [34] and Chen and Han [28], a yield criterion capable of capturing the typical anisotropic and sensitive to confining stress columnar-ice behavior at collapse is the following:

$a_{1}\left(\sigma_{y y}-\sigma_{z z}\right)^{2}+a_{2}\left(\sigma_{z z}-\sigma_{x x}\right)^{2}+a_{3}\left(\sigma_{x x}-\sigma_{y y}\right)^{2}+$

$+a_{4} \tau_{y z}^{2}+a_{5} \tau_{z x}^{2}+a_{6} \tau_{x y}^{2}+a_{7} \sigma_{x x}+a_{8} \sigma_{y y}+a_{9} \sigma_{z z}=1$ 
IJMS-08-37 Revised version R2.

where $a_{i}$ are nine material parameters necessary to define adequately the strength with respect to three mutually orthogonal planes of symmetry.

When restrictions due to plane strain condition are applied to ( 19 ), the following failure criterion is obtained (see [30][35] for details):

$a_{6}\left[\frac{\left(\sigma_{x x}-\sigma_{y y}\right)^{2}}{4}+\tau_{x y}^{2}\right]+\left(2 a_{7}+a_{9}\right) \frac{\left(\sigma_{x x}-\sigma_{y y}\right)}{2}-\left(1+\frac{a_{9}^{2}}{8 a_{1}}\right)=0$

where the restriction $a_{6}=2\left(a_{1}+2 a_{3}\right)$ due to symmetry in the material strength properties has to be taken into account.

The kinematical admissible velocities discontinuities corresponding to this yield criterion are nontrivial. Here, the formulation proposed by Krabbenhoft et al. in [34] is adopted, with a piecewise linearization with 30 segments of the failure surface in the $\sigma-\tau$ interface plane. In particular, the interface is considered as a degeneracy of two triangular elements with an edge of length $\delta \rightarrow 0$ as shown in Figure 20.

The velocity field in the local $\mathbf{t}-\mathbf{n}$ coordinate system for the triangle $T$ is defined as:

$u_{n}=\xi_{1} u_{n}^{1}+\xi_{2} u_{n}^{2}+\xi_{3} u_{n}^{3}$

$u_{t}=\xi_{1} u_{t}^{1}+\xi_{2} u_{t}^{2}+\xi_{3} u_{t}^{3}$

where $\xi_{i}$ are well known area coordinates (see for instance Sloan and Kleeman [1]), $u_{n}$ and $u_{t}$ are normal and tangential element velocities and $u_{t, n}^{i}$ are nodal velocities.

Differentiation of equation ( 21 ) gives the plastic strain rates in each element:

$\dot{\varepsilon}_{t t}=\frac{1}{L}\left(u_{t}^{2}-u_{t}^{3}\right)=\dot{\lambda} \frac{\partial f}{\partial \sigma_{t t}}$

$\dot{\gamma}_{t n}=\frac{1}{\delta}\left(u_{t}^{3}-u_{t}^{1}\right)=\dot{\lambda} \frac{\partial f^{t t}}{\partial \sigma_{t n}}$

$\dot{\varepsilon}_{n n}=\frac{1}{\delta}\left(u_{n}^{3}-u_{n}^{1}\right)=\dot{\lambda} \frac{\partial f}{\partial \sigma_{n n}}$

The following remarks may be done on the previous expression:

1. $\dot{\varepsilon}_{t t}=0$, being non degenerated elements rigid and infinitely strong $\left(u_{t}^{2}=u_{t}^{3}\right)$; 
2. the jump of displacements vector $\Delta \mathbf{u}^{1}$ on node 1 of the interface are defined as:

$$
\Delta \mathbf{u}^{1}=\left[\begin{array}{c}
\Delta u_{n}^{1} \\
\Delta u_{t}^{1}
\end{array}\right]=\left[\begin{array}{c}
\dot{\gamma}_{t n} \delta \\
\dot{\varepsilon}_{n n} \delta
\end{array}\right]=\left[\begin{array}{c}
\left.u_{t}^{3}-u_{t}^{1}\right) \\
u_{n}^{3}-u_{n}^{1}
\end{array}\right]=\left[\begin{array}{c}
\dot{\Gamma} \frac{\partial f}{\partial \sigma_{t n}} \\
\dot{\Gamma} \frac{\partial f}{\partial \sigma_{n n}}
\end{array}\right]
$$

where $\dot{\Gamma}$ is the plastic multiplier rate of the element integrated along the infinitesimal thickness of the interface.

The condition $\dot{\varepsilon}_{t t}=0$ of the elements belonging to the interface, via the associated flow rule imposition, leads to a further equality constraint for each node of each interface, which reads as follows:

$\dot{\varepsilon}_{t t}=\frac{1}{L}\left(u_{t}^{2}-u_{t}^{3}\right)=0 \Rightarrow \dot{\lambda} \frac{\partial f}{\partial \sigma_{t t}}=0 \Rightarrow \frac{\partial f}{\partial \sigma_{t t}}=0$

Following the ice indentation experiments conducted by Ralston [35], parameters $a_{1}, a_{3}, a_{7}$ and $a_{9}$ are assumed as follows:

$a_{1}=\frac{1.77}{C_{x}^{2}} \quad a_{3}=\frac{5.27}{C_{x}^{2}} a_{7}=\frac{6.04}{C_{x}} a_{9}=-\frac{3.54}{C_{x}}$

where $C_{x}$ is the in-plane unconfined compressive strength (here assumed equal to 1 for the sake of simplicity).

For the simulations, the initial mesh of Figure 21 has been used. The results obtained in terms of failure load are reported at successive iterations in Figure 22, together with the analytical upper bound found by Reinicke and Ralston [30] and the upper bound solution by Krabbenhoft et al. [34]. The initial mesh failure mechanism is shown in Figure 23, whereas in Figure 24 the final curved mesh with the corresponding failure mechanism are depicted. As it is possible to argue from Figure 22 , the final mesh gives very satisfactory results in terms of collapse load, even furnishing a solution more accurate than that proposed by Reinicke and Ralston [30] (see also [28]). As in the previous case, the advantage of the utilization of curved elements is evident. Results in this case are in discrete agreement with those obtained by Krabbenhoft et al. [34]. Despite the fact that in [34] a 
IJMS-08-37 Revised version R2.

lower value of the collapse load is obtained, it should be mentioned that results reported in [34]

were obtained with a very refined mesh and that the percentage difference with respect to present results is around $2 \%$, meaning that the present procedure can be used for technical purposes.

\subsection{Application to a masonry wall}

In the final example, the capabilities of the model are assessed by means of the limit analysis (see Figure 25) of a clay masonry shear wall tested by Ganz and Thürlimann [31] at ETH Zurich and analysed in Lourenço [36]. Experimental evidences show a very ductile response, so justifying the use of limit analysis for predicting the collapse load, with tensile and shear failure along diagonal stepped cracks.

A homogenization approach is used for the analyses, based on the simple equilibrated limit analysis micro-mechanical model proposed by Milani et al. [32][37]. Thus, the heterogeneous model composed by bricks and mortar joints, is substituted by a fictitious homogeneous material obtained through micro-mechanical considerations performed at the micro-scale, once that a suitable boundary values problem on the elementary cell (which generates the wall by repetition) is solved. For joints, a linearized Lourenço and Rots [38] failure criterion in adopted, able to gather typical frictional failure of joints, tensile failure and compressive crushing. Mechanical properties are summarized in Table I. For bricks, a Mohr-Coulomb failure criterion in plane stress is adopted (see Table I). Following the procedure presented in [32], a linearization with 58 planes representing a lower bound approximation of masonry failure surface $S^{\text {hom }}$ is utilized.

ETH Zurich shear tests are well suited for the validation of the model, not only because they are large and fit well with homogenization concepts, but also because most of the parameters necessary to characterize the model are available from biaxial tests [39]. Figure 25 shows the geometry of the wall considered here, which consist of a masonry panel of dimensions $3600 \times 2000 \times 150 \mathrm{~mm}^{3}$ $(L \times H \times t)$ and two flanges of dimensions $150 \times 2000 \times 600 \mathrm{~mm}^{3}\left(t \times H \times L_{f}\right)$, and the failure crack pattern experimentally obtained. Additional boundary conditions are given by two concrete slabs 
IJMS-08-37 Revised version R2.

placed in the top and bottom of the specimen. Initially, the wall is subjected to a vertical load $p$ uniformly distributed over the length of the wall with a resultant $P$. This is followed by the application of a horizontal force $F$ on the top slab along a horizontal displacement $d$.

A regular mesh of 160 linear or curved triangular elements is used for the present simulations, as shown in Figure 26. It is noted that the self-weight of the wall and the top slab are also considered in the analyses.

The properties of the composite material are obtained from Ganz and Thürlimann [39]. A low tensile strength value is assumed in the direction normal to the bed joints, which approximates well a no tension material model. It is noted that the flanges have the width of a single unit and the failure in the $x$ direction (equivalent to the out-of-plane direction of the panel) is determined by the tensile and compressive strength of the clay brick. Therefore, for the flanges new inelastic properties are assumed, namely tensile strength $f_{t}$ equal $0.68 \mathrm{~N} / \mathrm{mm}^{2}$ and compressive strength $f_{c}$ equal to $9.5 \mathrm{~N} / \mathrm{mm}^{2}$.

The wall analyzed here is denoted by $\mathrm{W} 1$ and is subjected to an initial vertical load $P$ of $415 k N$ corresponding to a moderately low vertical pressure equal to $0.61 \mathrm{~N} / \mathrm{mm}^{2}$. The wall shows a very ductile response with tensile and shear failure along the diagonal stepped cracks (see Figure 25-b). In Figure 26, a comparison between final and initial mesh failure mechanisms numerically obtained is reported. Authors experienced negligible differences between linear and curved elements performance during the optimization process, therefore only linear mesh failure mechanisms are reported for the sake of conciseness. It is particularly evident the good agreement between present numerical results and experimental evidences. The reader is also refereed to [40], where the same example has been analyzed with standard linear triangles with dissipation on both interfaces and elements (Sloan and Kleeman elements [1]), to confirm that present results fit well both experimental data and previously presented alternative numerical approaches. Finally, in Figure 27 the numerical collapse load at successive iterations obtained with both linear and curved elements is 
IJMS-08-37 Revised version R2.

represented. Experimental value of collapse load found in [31] (around $250 \mathrm{kN}$ ) is also depicted. As

it is possible to notice, collapse load evaluation confirms that both linear triangular and curved elements perform well with the example at hand, providing a converged upper bound shear strength for the wall (approximately $255 \mathrm{kN}$ ) very near to the experimental one.

\section{Conclusions}

A discontinuous upper bound limit analysis model with sequential linear programming mesh adaptation has been presented. In the model, rigid, infinitely strong triangular elements with both linear and Bezier curved edges have been used. A possible jump of velocities is allowed at the interfaces between contiguous elements, thus constraining plastic dissipation only at the interfaces. Bezier curved edges have been used with the sole aim of improving the element performance when dealing with limit analysis problems involving curved sliding surfaces. Since the model performs poorly for unstructured meshes (typically at the initial iteration), an iterative mesh adaptation based on the linearization of equality constraints (SLP) has been performed.

Several applications have been proposed, consisting in the determination of failure loads for a number of different problems (plates with holes, indentation problem on cohesive frictional soils, ice sheet indentation, masonry shear wall). Comparisons with literature show the reliability and efficiency of the method proposed from an engineering point of view. In order to evaluate the numerical performance of the method, a synopsis of CPU times required to obtain a converged solution and corresponding limit loads for all the examples analyzed are summarized in Table II. As it is possible to notice (also comparing present CPU times with data collected from the literature), the procedure proposed requires CPU times comparable with existing approaches. Nevertheless, despite the fact that fully rigorous and valuable alternatives (for instance based in the utilization of (a) adaptive NLP routines [10][13], (b) re-meshing techniques [9] and (c) p-FEM [29]) are already at disposal for the analysis at collapse of engineering problems, the procedure proposed has a number of advantages which makes it interesting for practitioners. The advantages are: (a) the 
IJMS-08-37 Revised version R2.

simplicity of the algorithm, which focuses exclusively on geometrical issues, (b) the robustness of the iterative approach, which requires only the recursive utilization of LP packages with few variables (usually available for free in the market) and (c) the very limited computational effort require for the optimization of real scale examples, strictly related to the utilization of splines elements (i.e. able to reproduce, in principle, complex curved failure mechanisms) with dissipation allowed only at the interfaces between contiguous elements. 


\section{References}

[1] Sloan SW, Kleeman PW (1995). Upper bound limit analysis using discontinuous velocity fields. Computer Methods in Applied Mechanics and Engineering 127:293-314.

[2] Lyamin AV, Sloan SW (2002). Lower bound limit analysis using non-linear programming, International Journal for Numerical Methods in Engineering 55: 573-611.

[3] Lyamin AV, Sloan SW (2002). Upper bound limit analysis using linear finite elements and non-linear programming, International Journal for Numerical and Analytical Methods in Geomechanics 26: 181-216.

[4] Krabbenhøft K, Damkilde L (2003). A general nonlinear optimization algorithm for lower bound limit analysis. International Journal for Numerical Methods in Engineering 56: $165-184$.

[5] Makrodimopoulos A, Martin CM (2007). Upper bound limit analysis using simplex strain elements and second-order cone programming. International Journal for Numerical and Analytical Methods in Geomechanics. 31(6): 835-865.

[6] Makrodimopoulos A, Martin CM (2007). Lower bound limit analysis of cohesivefrictional materials using second-order cone programming. International Journal for Numerical Methods in Engineering, 66(4): 604-634.

[7] Makrodimopoulos A, Martin CM (2007). Upper bound limit analysis using discontinuous quadratic displacement fields. Communications in Numerical Methods in Engineering, in press.

[8] Christiansen E, Andersen KD (1999). Computation of collapse loads with von Mises type yield condition. International Journal for Numerical Methods in Engineering 45: 11851202.

[9] Christiansen E, Pedersen OS (2001). Automatic mesh refinement in limit analysis. International Journal for Numerical Methods in Engineering 50: 1331-1346.

[10] Lyamin AV, Krabbenhøft K, Sloan SW, Hjiaj M (2004). An adaptive algorithm for upper bound limit analysis using discontinuous velocity fields. Proceedings of European Congress on Computational Methods in Applied Sciences and Engineering (ECCOMAS 2004), Jyväskylä, Finland.

[11] Chen S, Liu Y, Cen Z (2008). Lower bound shakedown analysis by using the element free Galerkin method and non-linear programming. Computer Methods in Applied Mechanics and Engineering 197: 3911-3921. 
[12] Ngo NS, Tin-Loi F (2007) Shakedown analysis using the p-adaptive finite element method and linear programming, Engineering Structures 29: 46-56.

[13] Lyamin AV, Sloan SW, Krabbenhoft K, Hjiaj M (2005). Lower bound limit analysis with adaptive remeshing. International Journal for Numerical Methods in Engineering 63: 1961-1974.

[14] Foley D, van Dam A, Feiner SK, Hughes JF (1996). Computer Graphics: Principles and Practice (2nd Edition). New York: Addison-Wesley.

[15] Ueshiba T, Roth G (1999). Generating smooth surfaces with bicubic splines over triangular meshes, 2nd International Conference on Recent Advances in 3D Imaging and Modelling, Ottawa, Canada, 302-311October.

[16] Piegl L, Tiller W (1997). The NURBS book. Springer, Berlin.

[17] Ferris M, Tin-Loi F. Limit analysis of frictional block assemblies as a mathematical program with complementarity constraints. Int. J. Mech. Sci. 2001; 43: 209-224.

[18] Matlab User's Guide, Version 6. The MathWorks, June 2001.

[19] Anderheggen E, Knopfel H (1972). Finite element limit analysis using linear programming. . International Journal of Solids and Structures 8: 1413-1431.

[20] Olsen PC (2001). Rigid-plastic finite element analysis of steel plates, structural girders and connections. Computer Methods in Applied Mechanics and Engineering 191: 761781.

[21] Sloan SW (1988). A steepest edge active set algorithm for solving sparse linear programming problems. International Journal Numerical Methods Engineering 12: 61-67.

[22] Poulsen PN, Damkilde L (2000). Limit state analysis of reinforced concrete plates subjected to in-plane forces. International Journal of Solids and Structures 37: 6011-6029.

[23] Johnson D (1995). Yield-line analysis by sequential linear programming. International Journal of Solids and Structures 32(10): 1395-1404.

[24] Ramsay ACA, Johnson D (1998). Analysis of practical slab configurations using automated yield-line analysis and geometric optimization of fracture pattern. Engineering Structures 20(8): 647-654.

[25] Johnson D (2001). On the safety of the strip method for reinforced concrete slab design. Computers and Structures 79: 2425-2430.

[26] Johansen KW (1962). Yield-line theory. Cement and Concrete Association, London.

[27] Strand 7.2 User's manual. www.strand7.com

[28] Chen WF, Han DJ. Plasticity for structural engineers. Springer, New York 1988. 
IJMS-08-37 Revised version R2.

[29] Tin-Loi F, Ngo NG. Performance of the p-version finite element method for limit analysis. International Journal of Mechanical Sciences 2003; 45: 1149-1166.

[30] Reinicke KM, Ralston TD (1977). Plastic limit analysis with an anisotropic yield function. International Journal of Rock mechanics and Mining Science 14: 147-154.

[31] Ganz HR, Thürlimann B (1984). Tests on masonry walls under normal and shear loading (in German). Report No. 7502-4. Institute of Structural Engineering, ETH Zurich, Zurich, Switzerland.

[32] Milani G, Lourenço PB, Tralli A (2006). Homogenised limit analysis of masonry walls. Part I: failure surfaces. Computers and Structures 84(3-4): 166-180.

[33] Yu X, Tin-Loi F (2006). A simple mixed finite element for static limit analysis. Computers and Structures 84: 1906-1917.

[34] Krabbenhoft K, Lyamin AV, Hjiaj M, Sloan SW (2005). A new discontinuous upper bound limit analysis formulation. International Journal for Numerical Methods in Engineering 63: 1069-1088.

[35] Ralston TD (1978). An analysis of ice sheet indentation. In: 4th International Symposium on Ice Problems. International Association for Hydraulic Research: Lulea, Sweden: 1331.

[36] Lourenço PB (1996). Computational strategies for masonry structures. Ph. Thesis, Delf University of Technology

[37] Milani G, Lourenço PB, Tralli A (2006). Homogenised limit analysis of masonry walls. Part II: structural examples. Computers and Structures 84(3-4): 181-195.

[38] Lourenço PB, Rots J (1997). A multi-surface interface model for the analysis of masonry structures. Journal of Engineering Mechanics ASCE, 123(7): 660-668.

[39] Ganz HR, Thürlimann B (1982). Tests on the biaxial strength of masonry (in German). Report No. 7502-3, Institute of Structural Engineering, ETH Zurich, Zurich, Switzerland.

[40] Lourenço PB, Milani G, Zucchini A, Tralli A (2007). The analysis of masonry structures: review and possibilities of homogenisation techniques. Canadian Journal of Civil Engineering, 34(11): 1443-1457. 


\section{Figures}
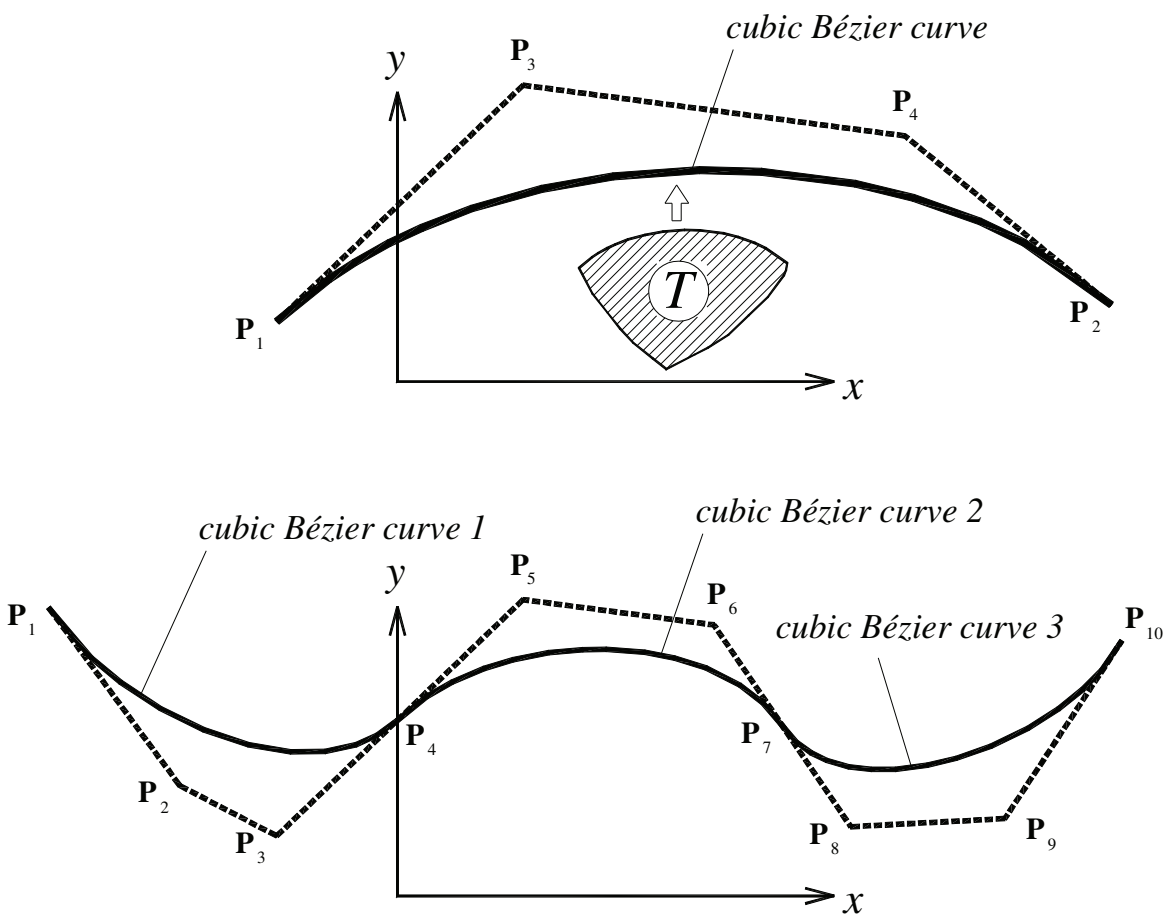

Figure 1: Typical cubic Beziér curve with the identification of control points (-a) and C1 continuity between two contiguous curves (-b).

Curved element

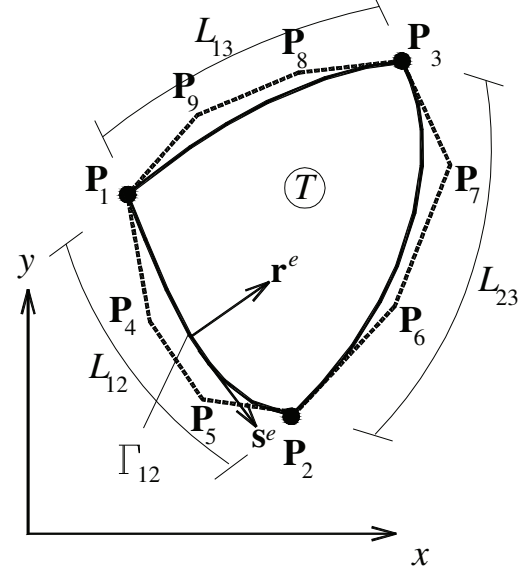

Figure 2: Curved and linear triangular elements used for the simulations. 


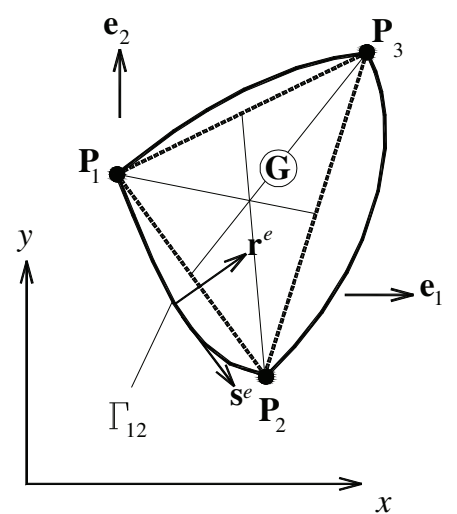

-a

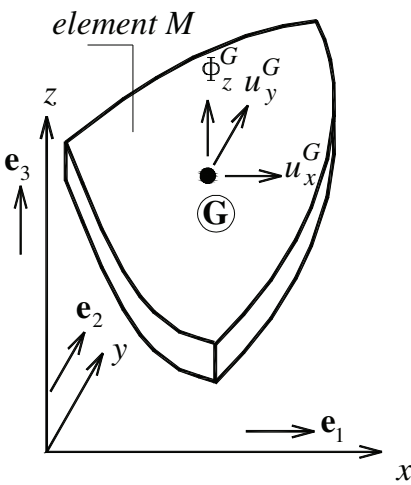

-b

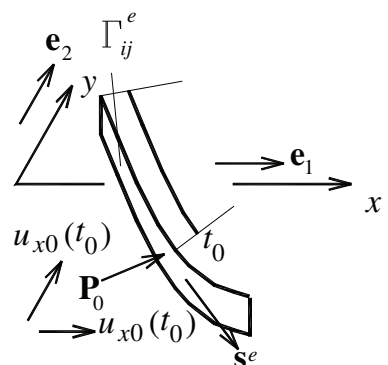

-c

Figure 3: Generic curved triangular element. -a: G coordinates identification. -b: optimization variables involved for an infinitely resistant element. -c: Local coordinates of a generic point on the edge.

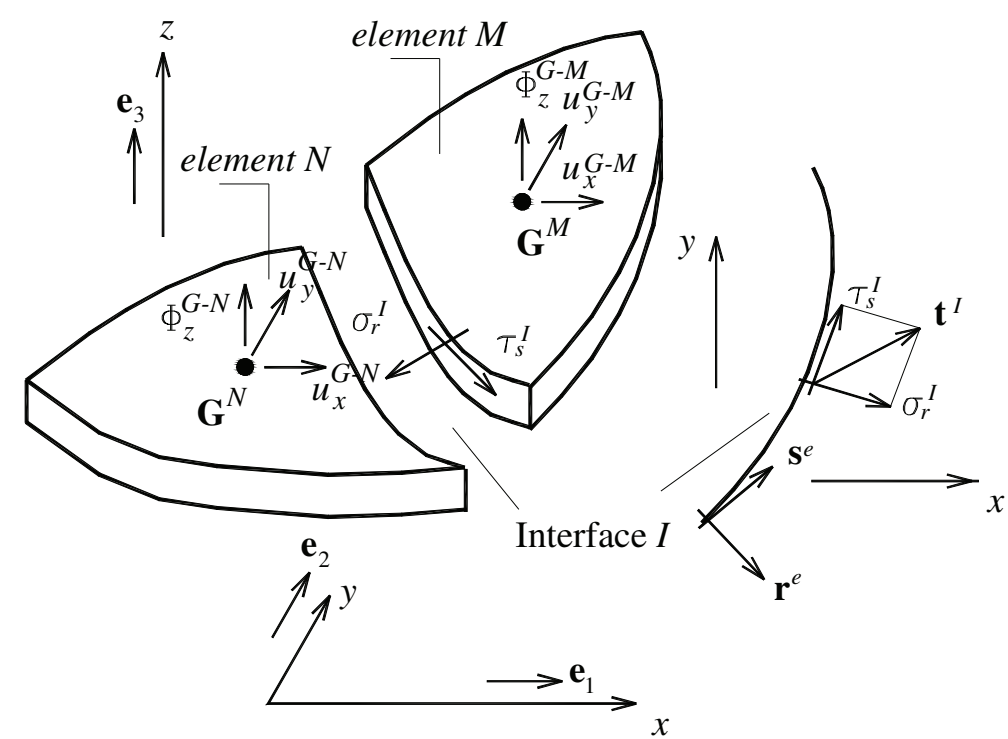

Figure 4: Generic curved interface between contiguous elements. $\mathbf{t}^{I}$ is a stress vector acting at the interface $I$, $\sigma_{r}^{I}$ is the stress component along local axis $\mathbf{r}^{e}$ and $\tau_{s}^{I}$ the stress component along $\mathbf{s}^{e}$. 


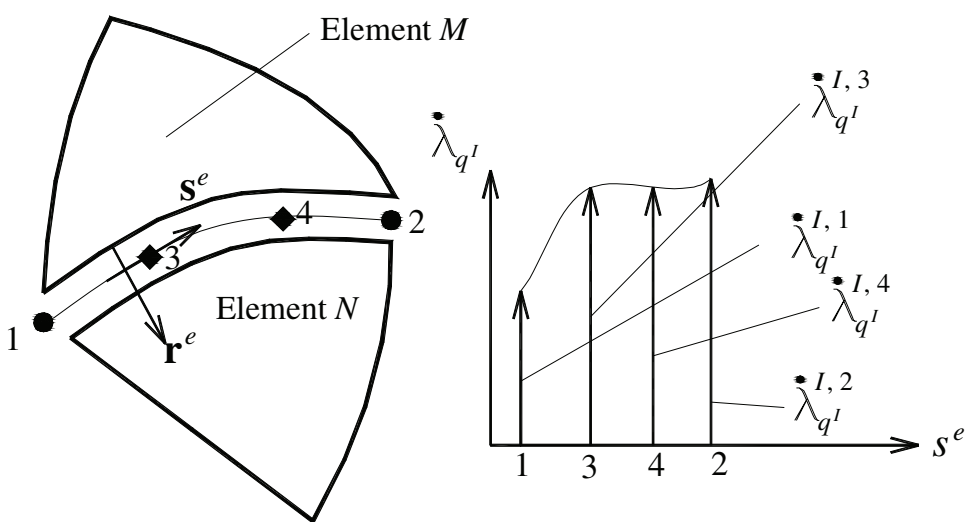

Figure 5: Plastic multipliers field interpolation in a generic interface between two contiguous elements.

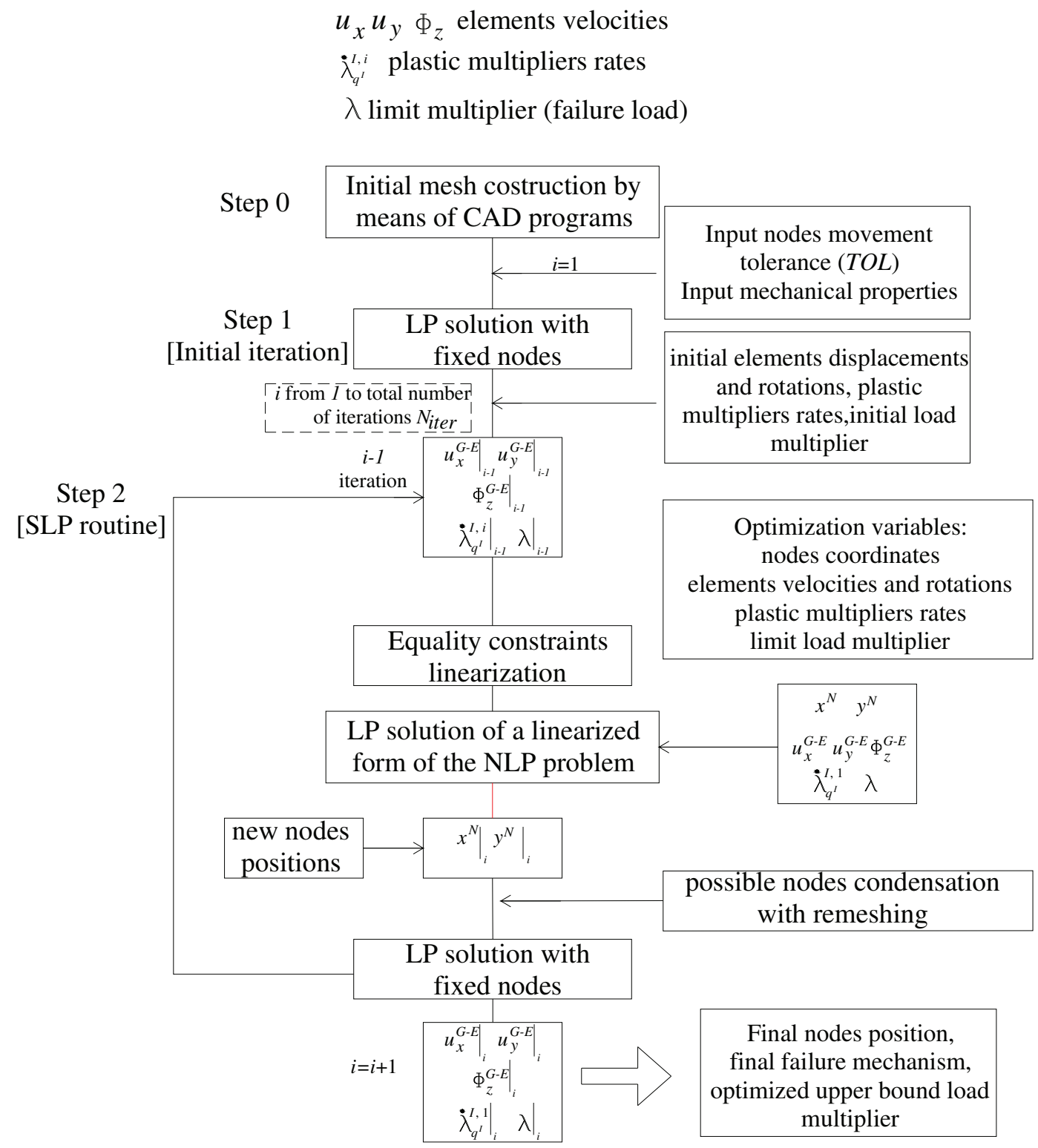

Figure 6: Sequential linear programming (SLP) adopted pseudo-code. 

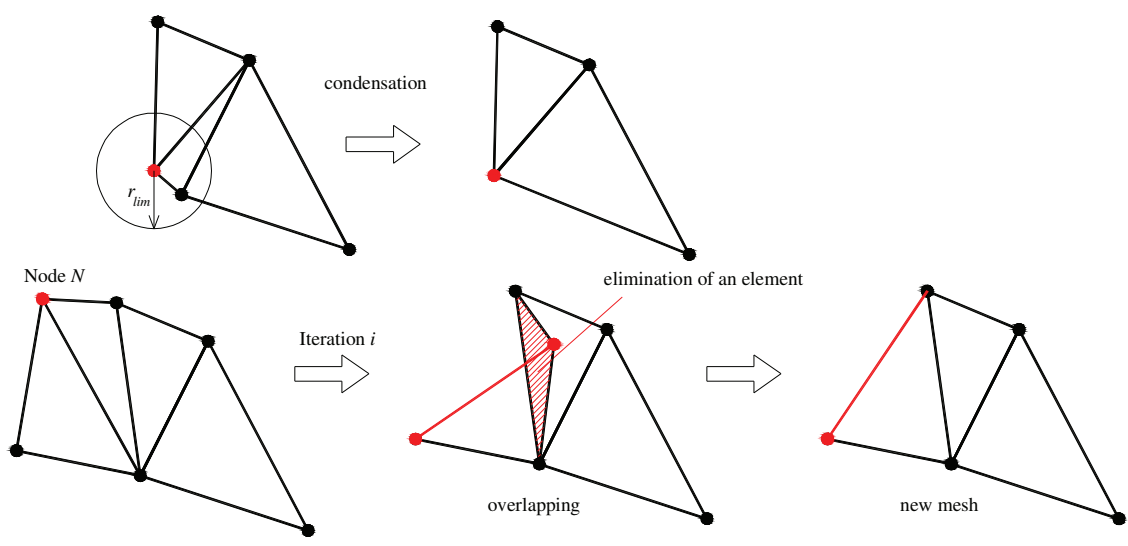

$-b$

Figure 7: Strategy adopted at the end of each iteration in order to avoid topological errors. -a: nodes condensation. -b: elimination of an element and remeshing.

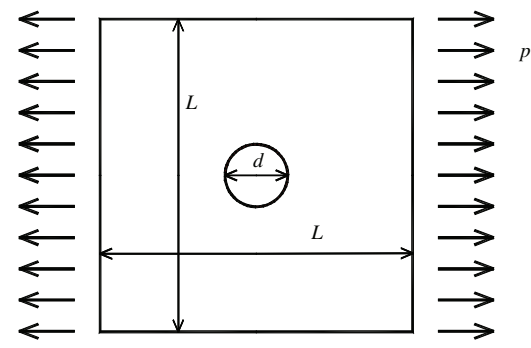

Figure 8: square plate with central circular hole, geometry
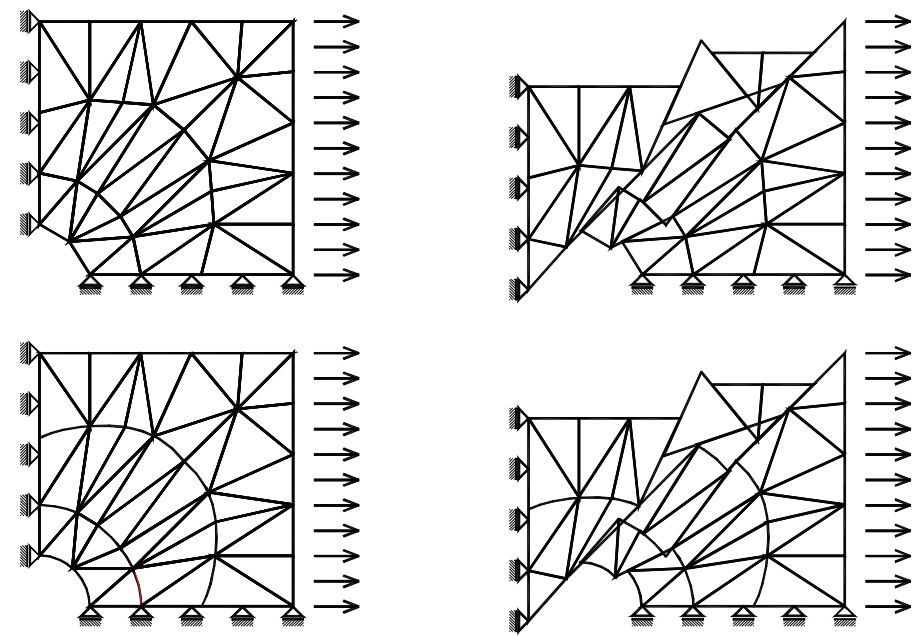

$-\mathbf{b}$

Figure 9: square plate with central circular hole, initial mesh (1/4 of the plate is meshed for symmetry) failure mechanism. -a: triangular three nodes element. -b: splines elements. 
IJMS-08-37 Revised version R2.
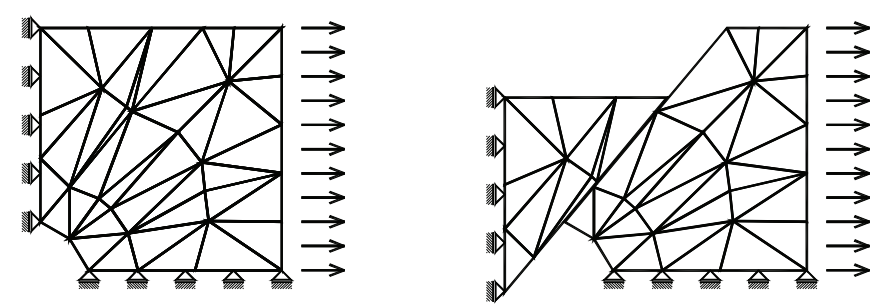

$-\mathbf{a}$
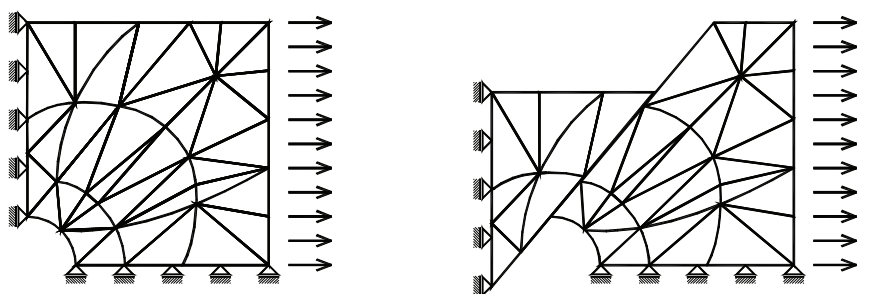

$-b$

Figure 10: square plate with central circular hole, final mesh (1/4 of the plate is meshed for symmetry) failure mechanism. -a: triangular three nodes element. -b: splines elements.

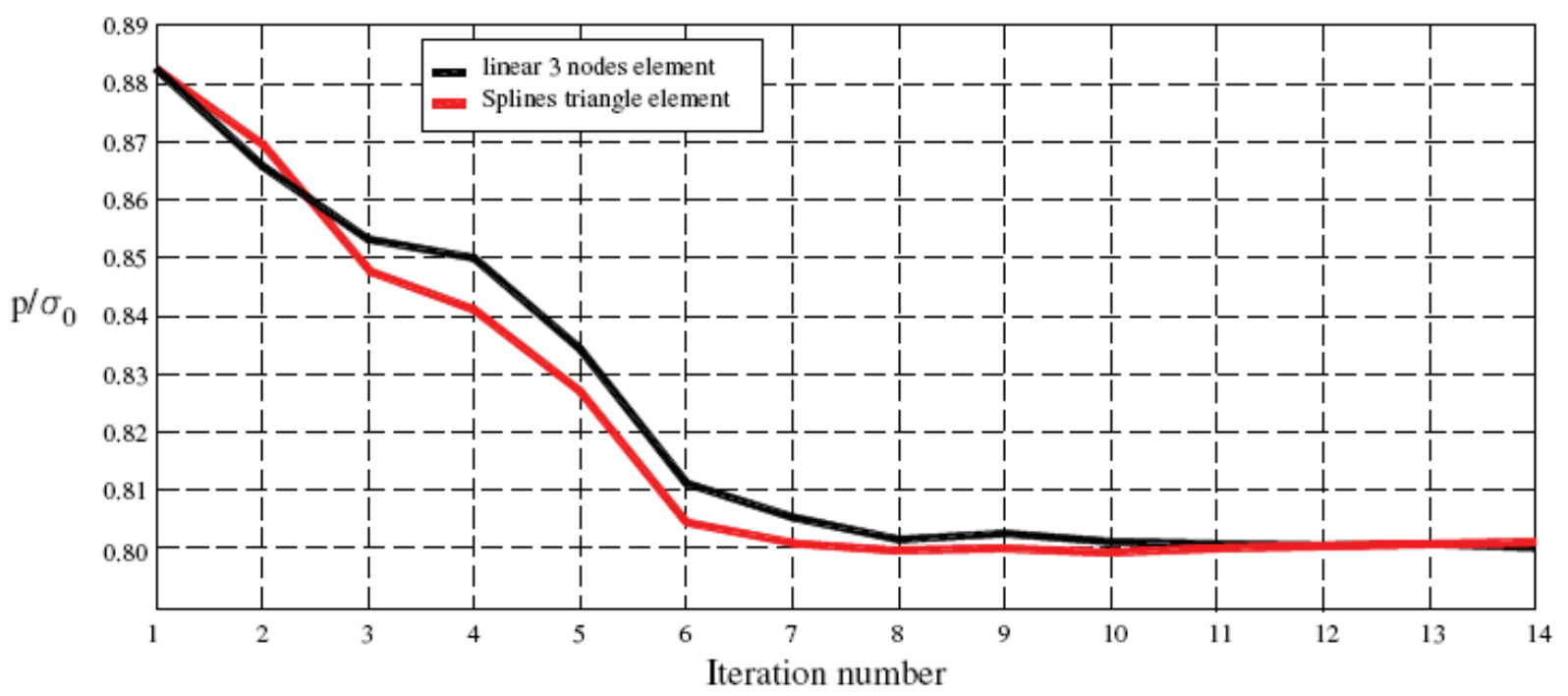

Figure 11: square plate with central circular hole, convergence of limit multiplier at successive iterations 
IJMS-08-37 Revised version R2.
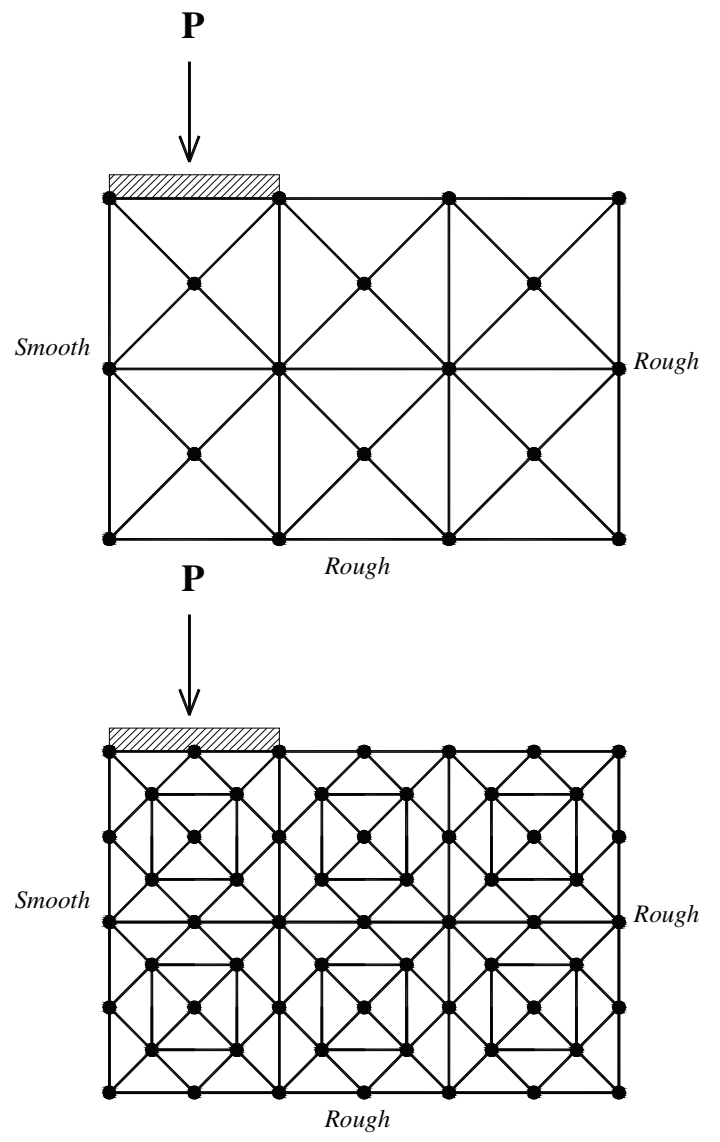

Figure 12: strip footing problem with Tresca failure surface. M1 mesh (triangular three nodes elements) and M2 Mesh (splines elements). 
IJMS-08-37 Revised version R2.

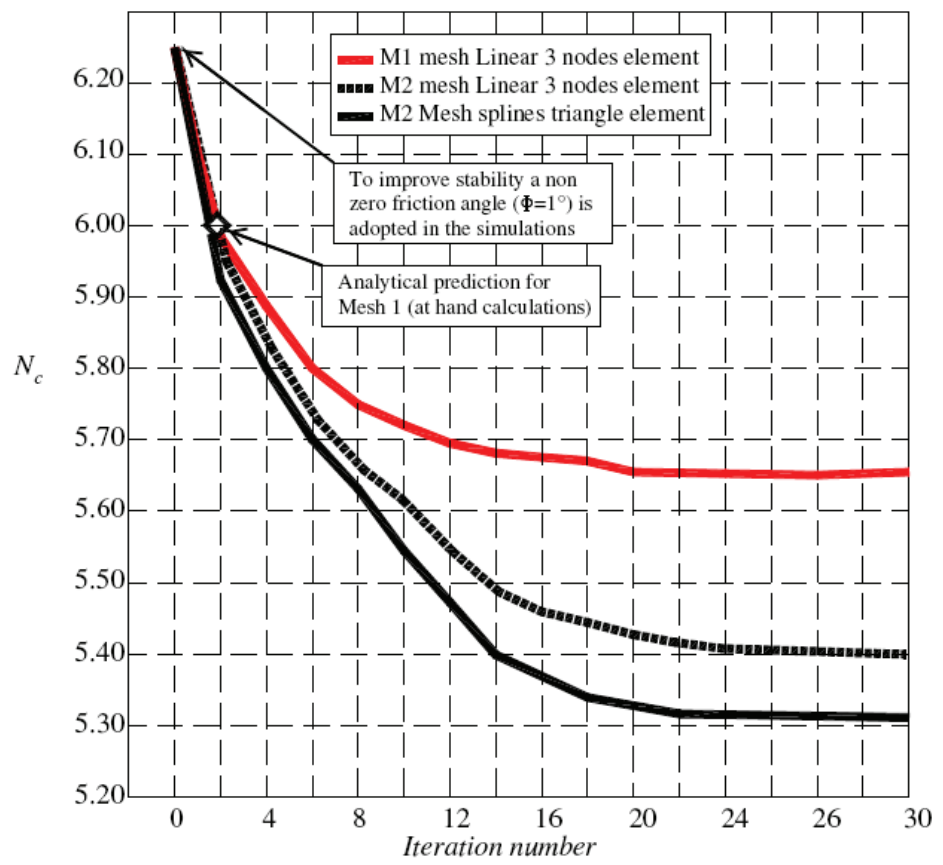

Figure 13: strip footing problem with Tresca failure surface. Sequential linear programming evaluation of $N_{c}$.

Iteration 1

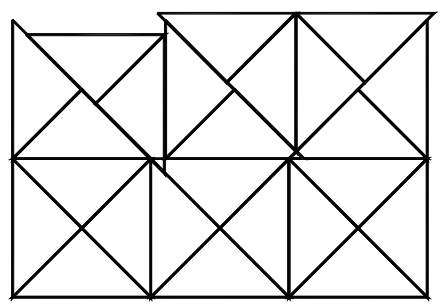

Iteration 4

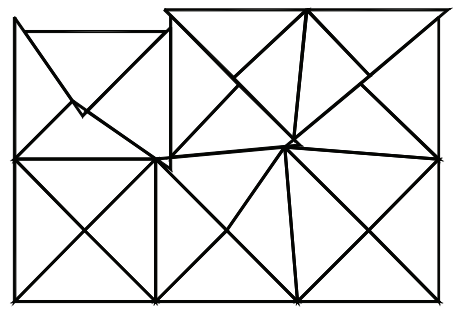

Iteration 12

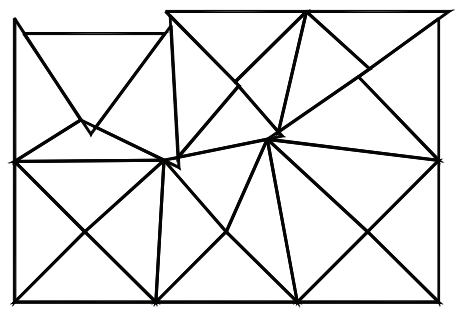

Figure 14: strip footing problem with Tresca failure surface, Mesh 1 at successive iterations. 
IJMS-08-37 Revised version R2.
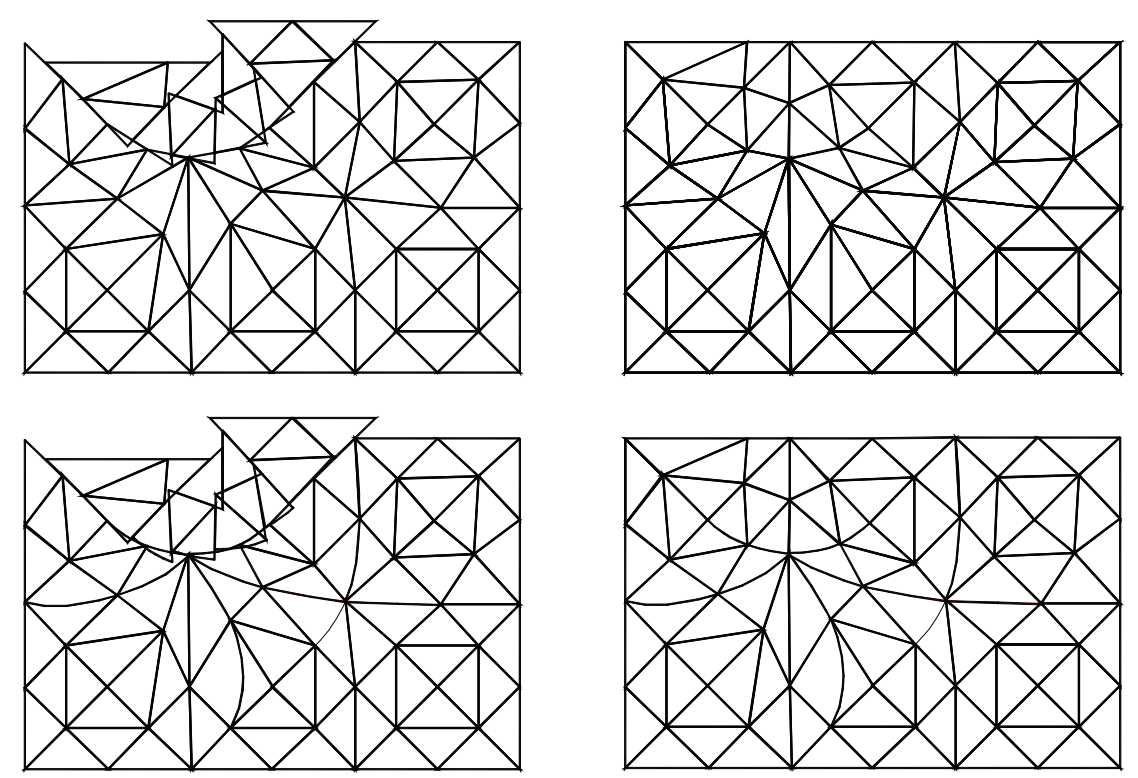

Figure 15: strip footing problem with Tresca failure surface. -a: final mesh M2, linear elements. -b: final mesh M2, splines elements.

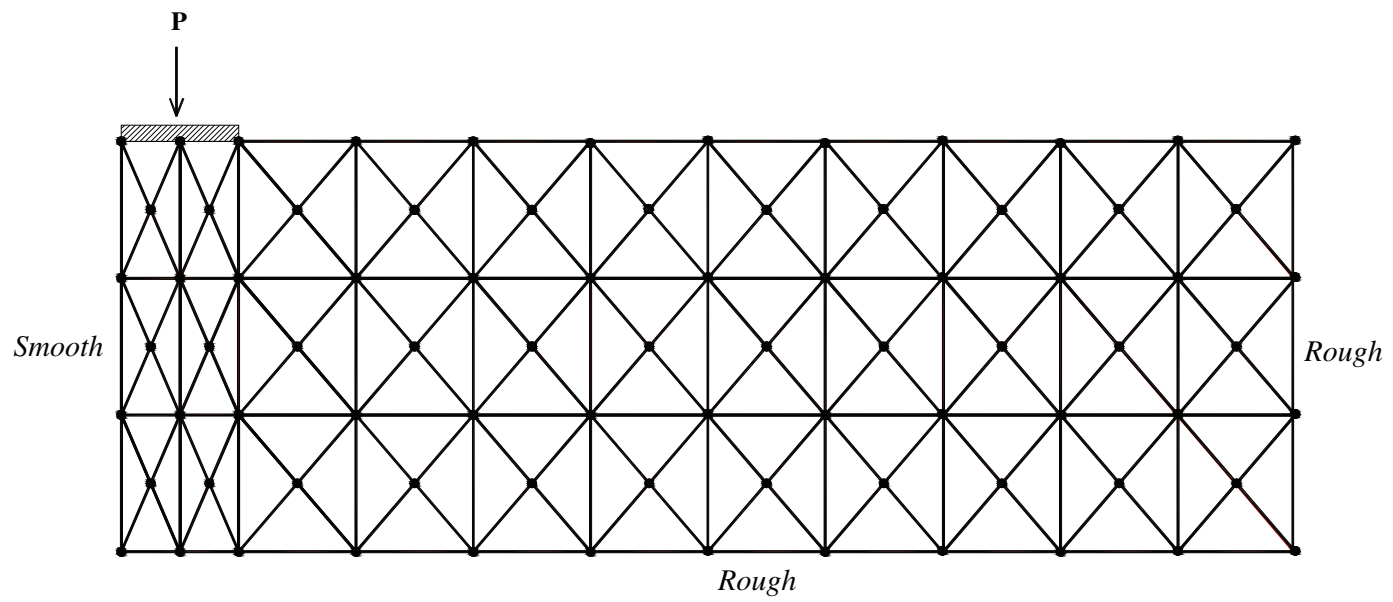

Figure 16: strip footing problem for a cohesive-frictional material. M1 mesh (triangular three nodes elements and splines elements). 
IJMS-08-37 Revised version R2.

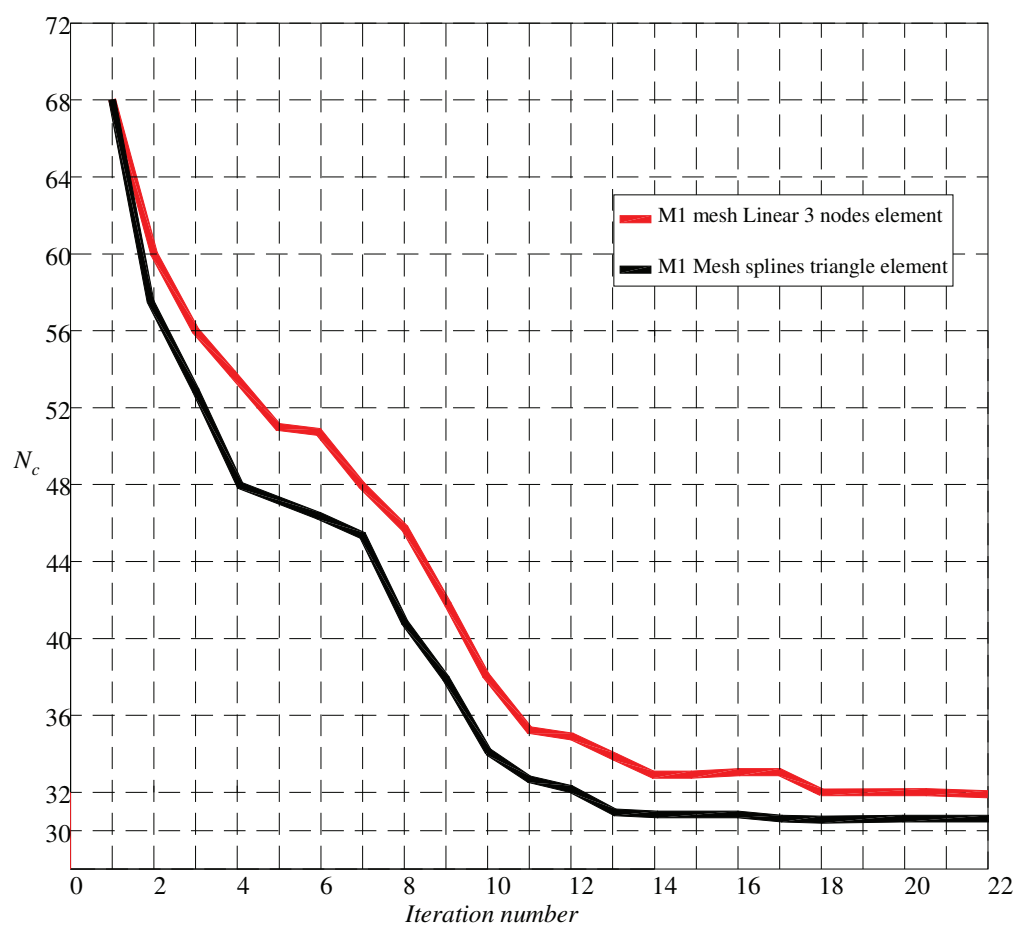

Figure 17: strip footing problem for a cohesive-frictional material. Sequential linear programming evaluation of $N_{c}$. 

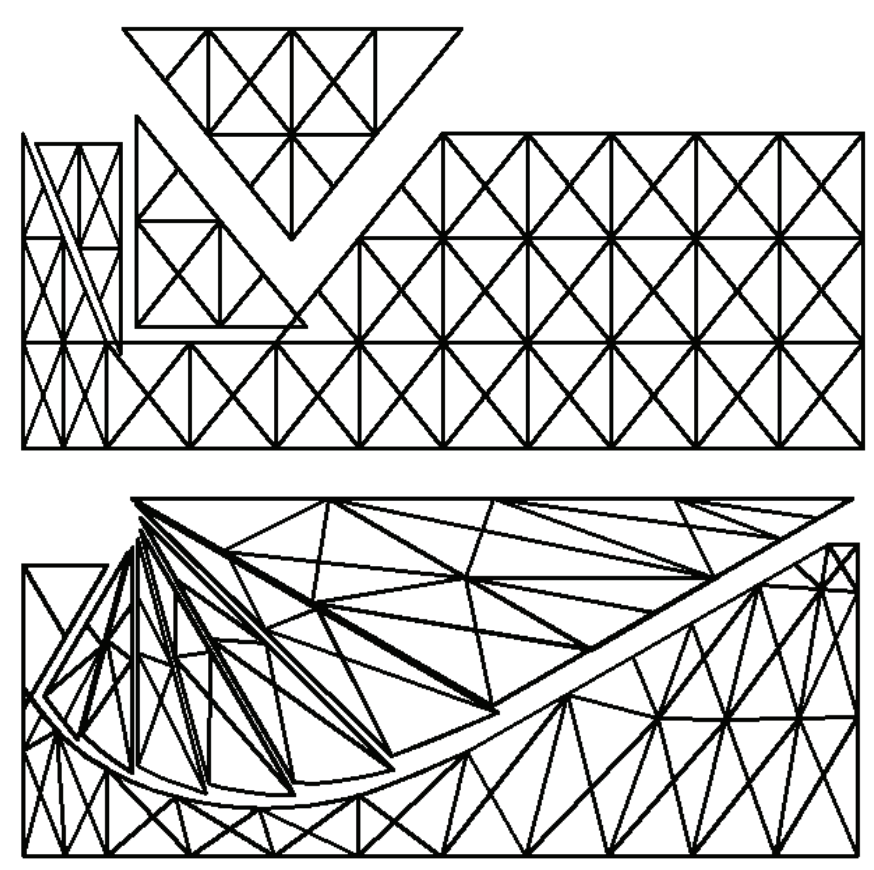

Figure 18: strip footing problem for a cohesive-frictional material. Adaptation of the mesh at successive iterations, first and last iteration.

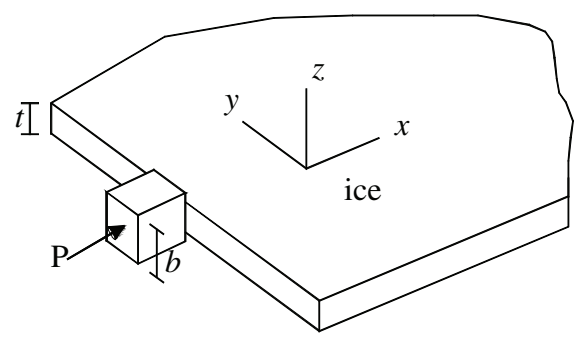

Figure 19: ice sheet indentation-physical problem. 
IJMS-08-37 Revised version R2.
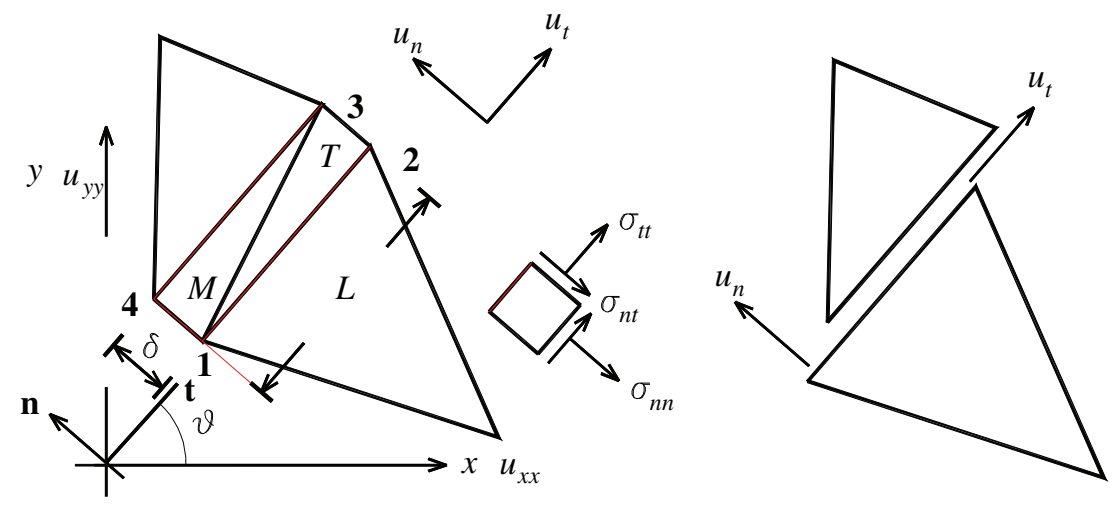

Figure 20: interpretation of interface elements when a piecewise linear approximation of the failure surface is used for the interfaces.

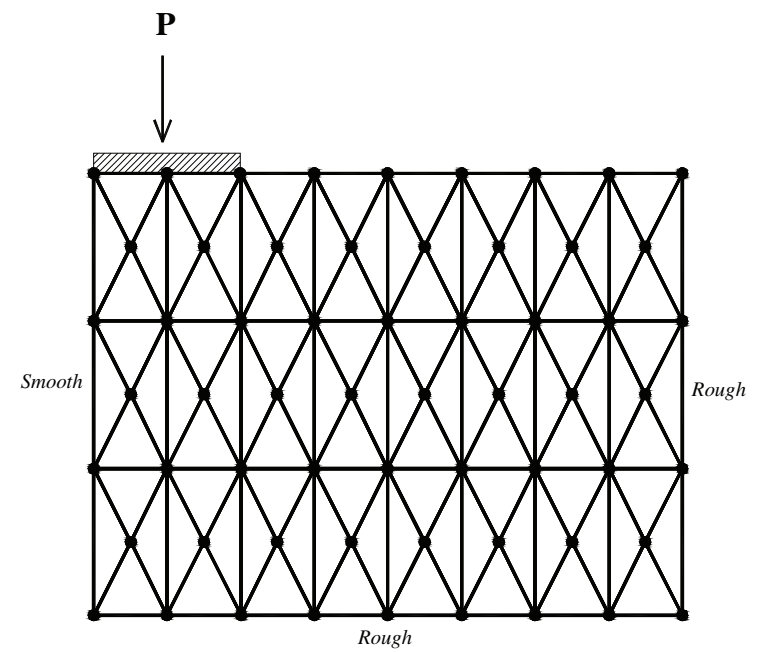

Figure 21: ice sheet indentation-physical problem, initial mesh M1 used for the simulations. 
IJMS-08-37 Revised version R2.

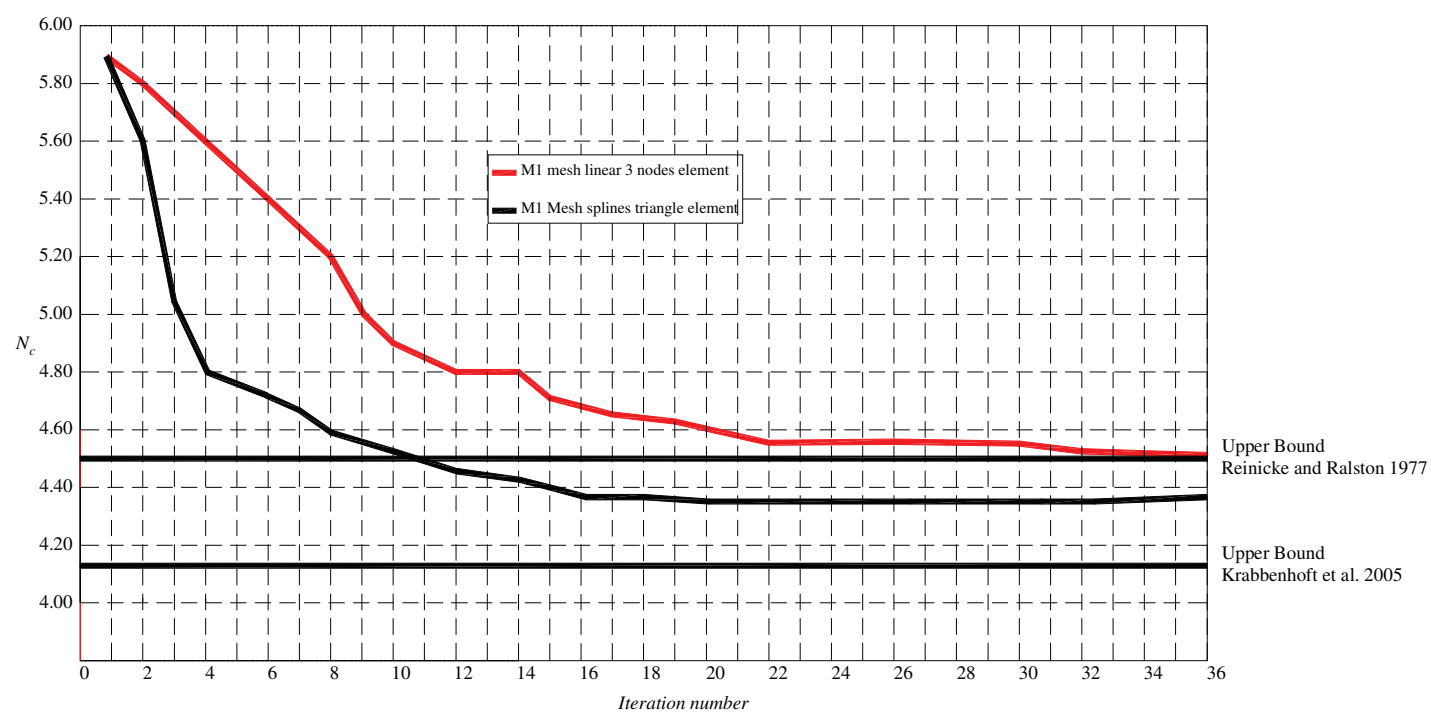

Figure 22: ice sheet indentation-physical problem. Sequential linear programming evaluation of failure load $N_{c}$. 
IJMS-08-37 Revised version R2.

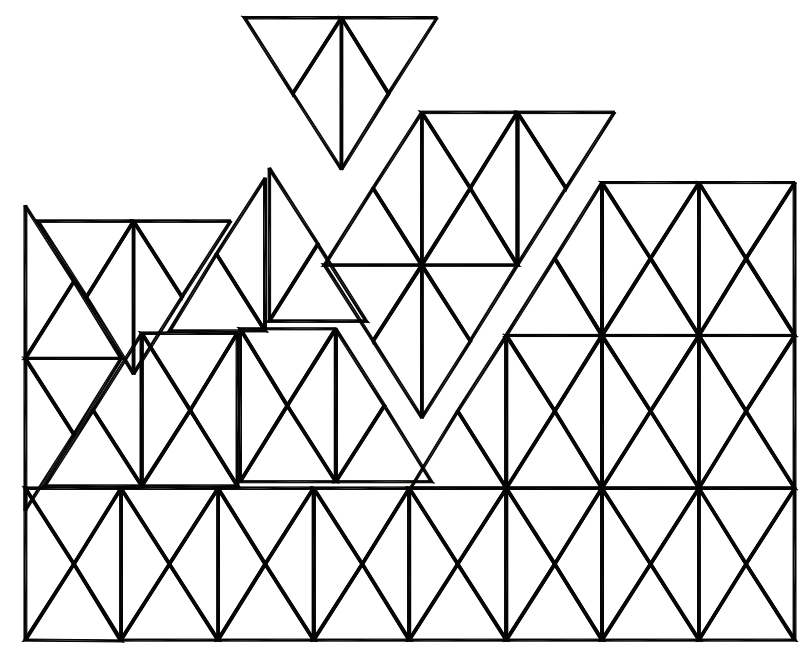

Figure 23: ice sheet indentation-physical problem, initial mesh failure mechanism.
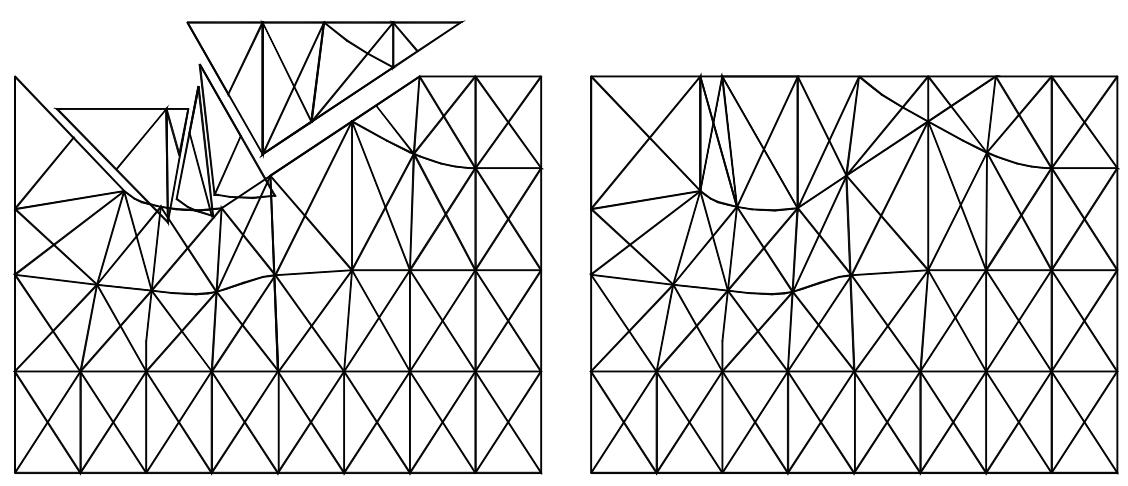

Figure 24: ice sheet indentation-physical problem, final mesh and failure mechanism, splines triangular element. 

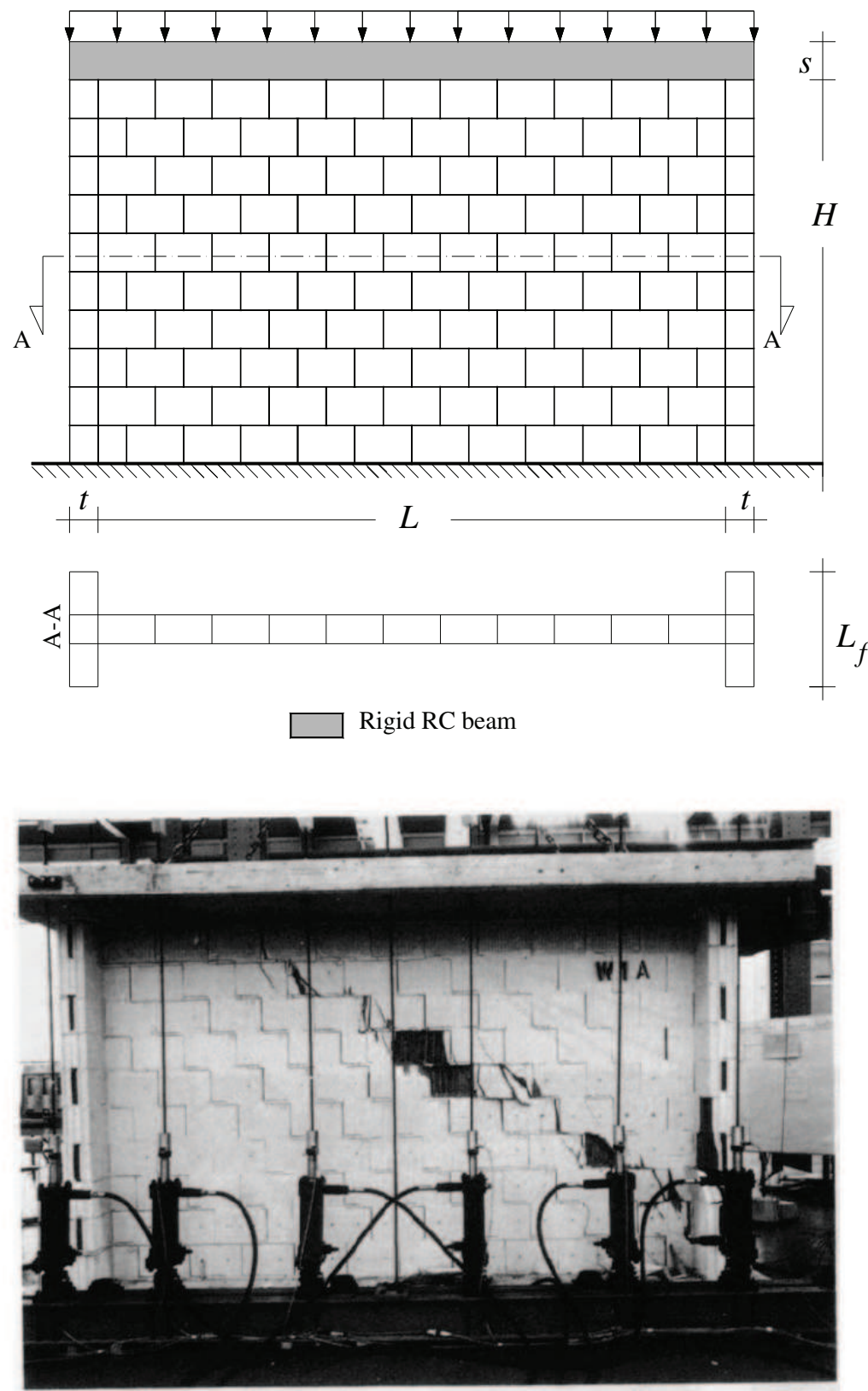

$-b$

Figure 25: geometry and loads for ETH Zurich shear walls (-a) and experimental failure mechanism (-b, from [31]). 

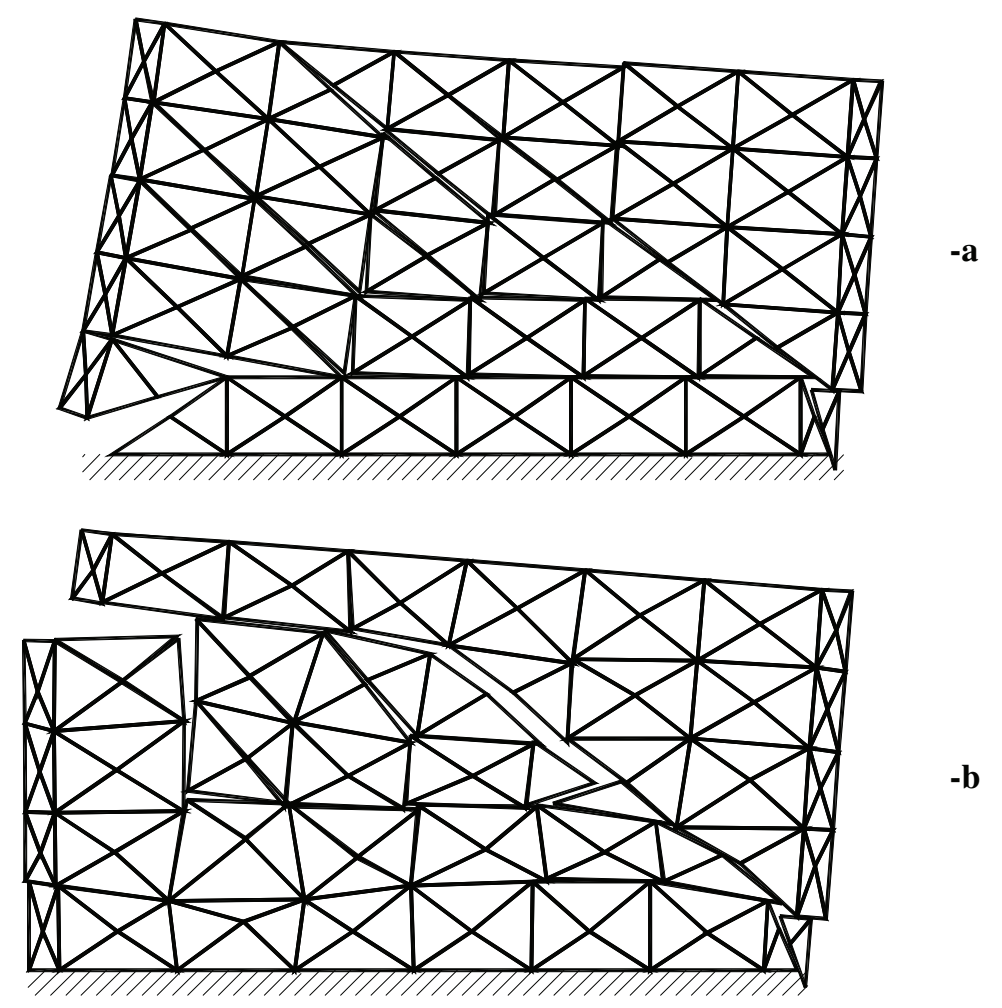

Figure 26: ETH Zurich shear wall, initial (-a) and final (-b) mesh failure mechanism (linear and splines elements provide almost the same results at the converged iteration, thus only linear elements are reported).

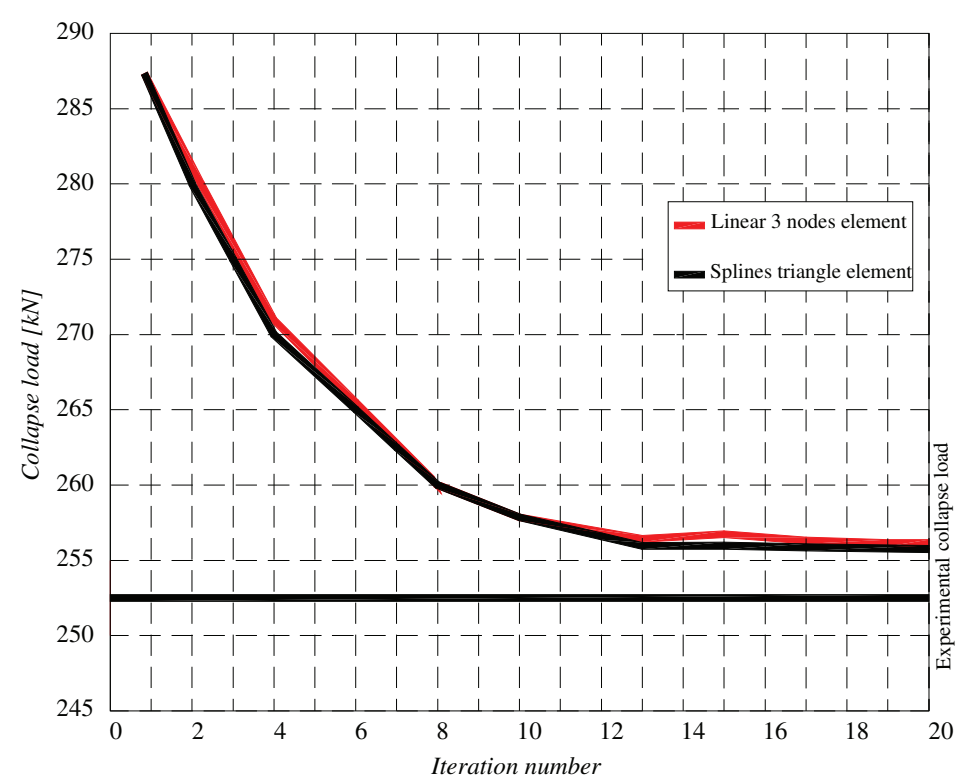

Figure 27: ETH Zurich shear test. Sequential linear programming evaluation of failure load. 
IJMS-08-37 Revised version R2.

\section{Tables}

Table I: ETH Zurich shear test. Mechanical characteristic assumed for joints and bricks.

\begin{tabular}{|c|c|c|}
\hline \multicolumn{3}{|c|}{ Joint (linearized Lourenço and Rots 1997 failure criterion) } \\
\hline$f_{t}\left[N / \mathrm{mm}^{2}\right]$ & Tensile strength & 0.05 \\
\hline$f_{c}\left[N / \mathrm{mm}^{2}\right]$ & Compressive strength & $4.74^{(*)}$ \\
\hline$c$ & Cohesion & $1.2 f_{t}$ \\
\hline$\Phi$ & Friction angle & $30^{\circ}$ \\
\hline$\Phi_{2}$ & Angle of the linerized compressive cap & $40^{\circ}$ \\
\hline \multicolumn{3}{|c|}{ Brick (Mohr-Coulomb failure criterion in plane stress) } \\
\hline \begin{tabular}{l|l}
$f_{c}$ & {$\left[N / \mathrm{mm}^{2}\right]$}
\end{tabular} & Compressive strength & 7 \\
\hline$\Phi$ & Friction angle & $45^{\circ}$ \\
\hline \multicolumn{3}{|c|}{ (*): the value adopted is evaluate as the mean of the horizontal and vertical compressive } \\
\hline
\end{tabular}


IJMS-08-37 Revised version R2.

Table II: CPU times required for the simulations reported in the paper and synopsys of the failure loads (PC used:MS OS, Intel Celeron 1.6 GHz processor, 1GB ram).

\begin{tabular}{|c|c|c|c|c|c|c|}
\hline \multirow[t]{2}{*}{ Example number } & \multicolumn{2}{|c|}{ Splines element } & \multicolumn{2}{|c|}{ Linear element } & \multicolumn{2}{|c|}{ Literature comparison } \\
\hline & LM & OT & LM & OT & LM & OT \\
\hline I: square plate with central hole & 0.80 & 67 & 0.80 & 59 & $\begin{array}{l}0.78^{(7)} \\
0.80^{(8)}\end{array}$ & $\begin{array}{l}0.2^{(7)} \\
618^{(8)}\end{array}$ \\
\hline $\begin{array}{l}\text { II: strip footing problem } \\
\text { (frictionless soil) }\end{array}$ & 5.31 & 91 & 5.40 & 78 & $\begin{array}{l}6.25^{(1)} \\
5.17^{(2)} \\
5.59^{(3)} \\
5.32^{(3)}\end{array}$ & $\begin{array}{c}0.1^{(1)} \\
2028^{(2)} \\
5.7^{(3)} \\
198.6^{(3)}\end{array}$ \\
\hline $\begin{array}{l}\text { III: strip footing problem } \\
\text { (friction soil) }\end{array}$ & 30.70 & 129 & 32.00 & 115 & $\begin{array}{c}37.26 \\
31.75 \\
\text { Error \% 8 } \\
\text { Error \% } 2.5^{(10)} \\
\text { Error \% } 16.2^{(11)} \\
\text { Error \% } 1.2^{(12)} \\
\end{array}$ & $\begin{array}{c}220^{(3)} \\
8906^{(3)} \\
1.82^{(9)} \\
13.6^{(10)} \\
0.60^{(11)} \\
5.0^{(12)} \\
\end{array}$ \\
\hline $\begin{array}{l}\text { IV: ice-sheet indentation } \\
\text { problem }\end{array}$ & 4.38 & 203 & 4.51 & 197 & $4.16^{(6)}$ & - \\
\hline V: ETHZ shear wall & $257^{(4)}$ & 509 & $257^{(4)}$ & 407 & $248^{(5)}$ & $312^{(5)}$ \\
\hline $\begin{array}{l}\text { LEGEND } \\
\text { LM Limit Multiplier } \\
\text { OT Optimization Time [sec] } \\
\text { (1) } \mathrm{p} \text {-FEM [29] with p=1 } \\
\text { (2) } \mathrm{p} \text {-FEM [29] with p=15 } \\
\text { (3) data from [1], with discontinu } \\
\text { (4) value expressed in kN } \\
\text { (5) data from [40] } \\
\text { (6) data from [34] } \\
\text { (7) data from [33], mixed elemen } \\
\text { (8) data from [33], mixed elemen } \\
\text { (9) data from [3], upper boun } \\
\text { collapse loads not possible (erro } \\
\text { (10) data from [3], upper bound, } \\
\text { loads not possible (error is calcu } \\
\text { (11) data from [2], lower boun } \\
\text { collapse loads not possible (erro } \\
\text { (12) data from [2], lower bound, } \\
\text { loads not possible (error is calcu }\end{array}$ & $\begin{array}{l}\text { coarse } n \\
\text { refined } \\
\text { example } \\
\text { is calcula } \\
\text { xample } \mathrm{k} \\
\text { ted with } \\
\text { exampl } \\
\text { is calcula } \\
\text { rample } \mathrm{W} \\
\text { tted with }\end{array}$ & $\begin{array}{l}\text { with } \\
\text { fricti } \\
\text { pect } t\end{array}$ & $\begin{array}{l}\text { ction ang } \\
\text { respect to } \\
\text { n angle } 3 \\
\text { Prandtl s } \\
\text { iction ang } \\
\text { respect to } \\
\text { n angle } 3 \\
\text { Prandtl s }\end{array}$ & $\begin{array}{l}35^{\circ}, \\
\text { randtl } \\
\text {, fine } \\
\text { lution) } \\
35^{\circ}, \\
\text { randtl } \\
\text {, fine }\end{array}$ & $\begin{array}{l}\text { coarse mesh. Con } \\
\text { solution) } \\
\text { mesh. Comparison } \\
\text { coarse mesh. Con } \\
\text { solution) } \\
\text { mesh. Comparison }\end{array}$ & $\begin{array}{l}\text { parison on } \\
\text { on collapse } \\
\text { parison on } \\
\text { on collapse }\end{array}$ \\
\hline
\end{tabular}

